\title{
BOUNDEDNESS OF DISPERSIVE DIFFERENCE SCHEMES
}

\author{
DONALD ESTEP, MICHAEL LOSS, AND JEFFREY RAUCH
}

\begin{abstract}
The pointwise behavior of dispersive difference schemes for the simple wave equation in one dimension is analyzed. If the initial data are in certain Besov spaces, the scheme is shown to be pointwise unbounded. Boundedness is shown when the initial data are of bounded variation.
\end{abstract}

\section{INTRODUCTION}

This paper is concerned with the pointwise behavior of constant-coefficient, single-step finite difference approximations to the solution of the initial value problem

$$
\begin{array}{ll}
u_{t}+u_{x}=0, & x \in \mathbf{R}, 0<t \leq T, \\
u(0, x)=v(x), & \dot{x} \in \mathbf{R},
\end{array}
$$

where $v$ is chosen in $L^{p}(\mathbf{R})$ for some $1 \leq p \leq \infty$. (Hereafter, we assume that $T=1$, since all the results generalize from this.) The (weak) solution of this problem is simply a translation of the initial data and the problem is well posed in every $L^{p}(\mathbf{R})$ space. This problem is considered to be a test case for numerical methods for the approximation of solutions of hyperbolic equations.

The approximant to the solution $u(t, x)$ at time $t=n k$, where $k$ denotes the time step, is called $U(n k, x)$. We let $E_{k}$ denote the time stepping operator associated with the scheme. Thus, the operator $E_{k}^{n}$ is the operator marching through a time interval $n k$, and we have

$$
U(n k, x)=E_{k} U((n-1) k, x)=E_{k}^{n} v(x) \text {. }
$$

In the case of an explicit scheme, such as the Lax-Friedrichs or Lax-Wendroff schemes, $E_{k}$ will be a finite linear combination of powers of a translation operator $\tau_{h}, h \in \mathbf{R}$, where $\left(\tau_{n} f\right)(x)=f(x-h)$ (see $\S 5$ ). An example of an implicit scheme for $u_{t}+u_{x}=0$ is the Crank-Nicolson scheme. Its operator is defined by

$$
\left(1+\frac{\lambda}{4} \tau_{-h}-\frac{\lambda}{4} \tau_{h}\right) E_{k} U(t, x)+\left(-1+\frac{\lambda}{4} \tau_{-h}-\frac{\lambda}{4} \tau_{h}\right) U(t, x)=0,
$$

Received April 20, 1989.

1980 Mathematics Subject Classification (1985 Revision). Primary 65M10.

The authors' research was partially supported by DARPA contract \#70NANB8H0860 (Estep), and U. S. National Science Foundation grants DMS-8801309 (Loss) and DMS-8601783 (Rauch). 
where $h>0$ is the spatial mesh width and $\lambda:=k / h$ is the mesh ratio. It is common to think of $x$ as being restricted to one of the mesh points $j h$, $j \in \mathbf{Z}$. In this case, $U(n k, j h)$ represents the approximation to the exact value $u(n k, j h)$ and the difference scheme is written

$$
\begin{aligned}
& \frac{U((n+1) k, j h)-U(n k, j h)}{k} \\
&+\frac{1}{2}\left\{\frac{U((n+1) k,(j+1) h)-U((n+1) k,(j-1) h)}{2 h}\right. \\
&\left.+\frac{U(n k,(j+1) h)-U(n k,(j-1) h)}{2 h}\right\}=0 .
\end{aligned}
$$

Remark. The term "difference" is used here in a general sense; many finite element approximation methods lead to time-stepping operators of this kind, and our discussion pertains to these schemes as well.

The primary object of the classical analysis of such methods is to prove convergence and error estimates for the scheme in various Banach spaces. According to the Lax Equivalence Theorem, the two principal ingredients are the consistency and stability of the scheme as measured in the norm of the space. Consistency is the requirement that the difference operator accurately model the solution operator of $(1.1)$ and is expressed by

$$
\frac{1}{k}\left\|u(t+k, \cdot)-E_{k} u(t, \cdot)\right\|=\mathrm{O}\left(k^{\mu-1}\right) \quad \text { as } k \rightarrow 0,
$$

for all smooth solutions $u$, where $\mu-1$ is the accuracy of the scheme. In what follows, we consider only difference schemes which are consistent with the equation in $(1.1)$ in all $L^{p}(\mathbf{R})$ norms. Stability is the requirement that the difference operator does not magnify error without bound. If $1 \leq p \leq \infty$, the difference scheme is said to be $L^{p}(\mathbf{R})$ stable if and only if there is a constant $C>0$ such that for all $v \in L^{p}(\mathbf{R})$ and $n k \leq 1$,

$$
\left\|E_{k}^{n} v\right\|_{L^{p}(\mathbf{R})} \leq C\|v\|_{L^{p}(\mathbf{R})} .
$$

The norms and spaces of initial data for which these two conditions are true vary from scheme to scheme and, in fact, a difference scheme is characterized exactly in this way. Of course, it is reasonable to require stability, hence convergence, in some space. We restrict this discussion to schemes which are stable in $L^{2}(\mathbf{R})$.

Actually, one can show that if a $L^{2}(\mathbf{R})$ stable difference scheme with $k / h$ constant is unstable in $L^{p}(\mathbf{R})$ for some $p \geq 1, p \neq 2$, then it is unstable in $L^{p}(\mathbf{R})$ for every $p, p \geq 1, p \neq 2$ (see [6]). Therefore, there is a natural separation of schemes into those which are stable in $L^{p}(\mathbf{R})$ for all $p \geq 1$ and those which are stable only in $L^{2}(\mathbf{R})$. The pointwise behavior of the former is well understood. An example is the Lax-Friedrichs method (see $\S 5$ ) which is a dissipative scheme. It is characteristic of dissipative schemes that initial data consisting of a step function undergo smoothing as time progresses.

We are interested in studying the pointwise behavior of schemes which are not $L^{\infty}(\mathbf{R})$ stable, i.e., the dispersive schemes such as the Lax-Wendroff and CrankNicolson methods (see [6]). The characteristic oscillations of the approximant 


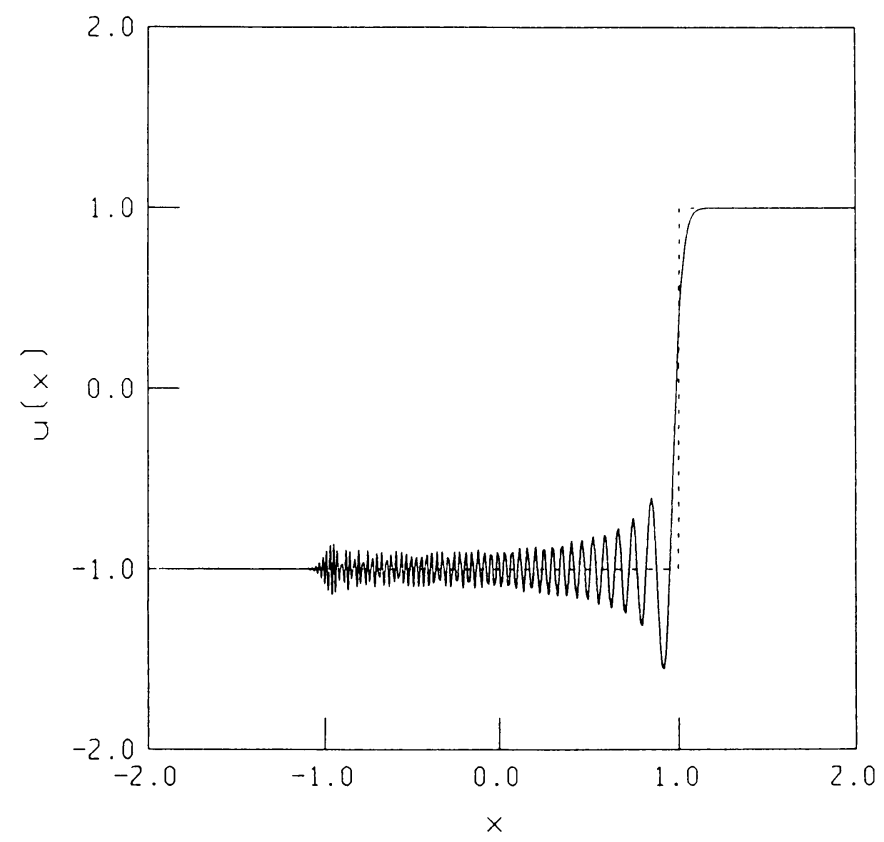

FIGURE 1.1

Plot of the Crank-Nicolson approximant computed on step data at $t=1, k=.01, \lambda=.5$. The dashes show the position of the true solution. The solid curve connects the points of the approximant.

in the region of the discontinuity of the true solution distinguishes these schemes (see §5). As an example, we present in Figure 1.1 the Crank-Nicolson scheme computed on the initial data $J(x):=\operatorname{sign}(x)$, the single step, using $k=.01$ and $\lambda=.5$.

It is useful to introduce the Fourier transform at this point. The time-stepping operator $E_{k}$ of a constant-coefficient finite difference scheme is a translation invariant operator of the form $E_{k}^{n}(D) v:=E_{k}^{n} v=\mathscr{F}^{-1}\left(E_{k}^{n}(\xi) \hat{v}(\xi)\right)$, where $\mathscr{F}$ denotes the Fourier transform with respect to the measure $d x:=d x / \sqrt{2 \pi}$. (This expression is to be interpreted in the sense of distributions for general $v \in L^{p}(\mathbf{R}), 1 \leq p \leq \infty$; see [6, Chapter 1].) In this notation, $E_{k}(\xi)$ is the symbol of the time-stepping operator $E_{k}(D)$ and our principal assumptions can be rephrased in terms of properties of the symbol of the scheme. First of all, consistency means that the symbol of the scheme satisfies

$$
E_{k}(\xi)=e^{-i k \xi+o(k|\xi|)} \quad \text { as } k|\xi| \rightarrow 0
$$

(see [6, Chapter 5]). As for $L^{p}(\mathbf{R})$ stability, it is identical to the condition that the family $\left\{E_{k}^{n}(D), n k \leq 1\right\}$ be bounded in $\operatorname{Hom}\left(L^{p}(\mathbf{R}), L^{p}(\mathbf{R})\right)$. 
It is well known that the set of $L^{2}(\mathbf{R})$ stable operators $a(D)$ consists precisely of those maps whose symbols are in $L^{\infty}(\mathbf{R})$ and that

$$
\|a(D)\|_{L^{2}(\mathbf{R}) \rightarrow L^{2}(\mathbf{R})}=\|a\|_{L^{\infty}(\mathbf{R})} .
$$

In what follows, we assume that the symbol of a difference scheme is at least continuous in $\xi$. Thus, a necessary and sufficient condition for the stability of a difference scheme in $L^{2}(\mathbf{R})$ is that there exists a $C>0$ with $\left|E_{k}(\xi)\right| \leq 1+C k$ for all $\xi \in \mathbf{R}, k \in(0,1)$. For example, the symbol of the Crank-Nicolson scheme is

$$
E_{k}(\xi)=\frac{1-i \lambda \sin (h \xi) / 2}{1+i \lambda \sin (h \xi) / 2} .
$$

In this case, $\left|E_{k}(\xi)\right| \equiv 1$, so $E_{k}(D)$ is $L^{2}(\mathbf{R})$ stable without condition. If $\lambda$ is constant, then it is known that $E_{k}(D)$ is stable in $L^{p}(\mathbf{R})$ only for $p=2$. (Note that the "overshoot" to the left of the step shows that this scheme is not a contraction on $L^{\infty}(\mathbf{R})$, but we show below that the "overshoots" are bounded as $k \rightarrow 0$.)

Since the 1960's, many authors have studied the stability of difference methods and the conditions which make a scheme dissipative or dispersive, including Apelkrans, Brenner, Chin, Hedstrom, Peetre, Thomée [1, 4, 5, 6, 8, 9, 12, 13, $18,22]$, and Trefethen [23, 24], who presented a nice overview of the subject and in the case of dispersive schemes made a connection to the classical theory of dispersion, see Whitham [26].

The analysis of the pointwise behavior of difference approximants becomes interesting in the case of schemes which are only $L^{2}(\mathbf{R})$ stable, and hence known only to converge in the $L^{2}(\mathbf{R})$ norm, because they represent a pointwise approximation to the true solution. In particular, $L^{\infty}(\mathbf{R})$ instability implies, thanks to the uniform boundedness principle, that there is a $v \in L^{\infty}(\mathbf{R})$ such that

$$
\sup _{n k \leq 1}\left\|E_{k}^{n}(D) v\right\|_{L^{\infty}(\mathbf{R})}=\infty
$$

On the other hand, everyday experience does not seem to produce such $v$. In particular, if $v$ is piecewise $C^{1}$, but possibly discontinuous, computations yield families which are uniformly bounded.

The first result we present classifies more precisely the space of initial data upon which dispersive difference schemes are unbounded. We say that the difference approximants are uniformly pointwise bounded if

for some constant $C$.

$$
\sup _{n k \leq 1}\left\|E_{k}^{n}(D) v\right\|_{L^{\infty}(\mathbf{R})} \leq C
$$

Our approach is suggested by the observation that an $L^{2}(\mathbf{R})$ stable scheme is also stable in the Sobolev spaces $H^{s}$. We ask if taking data in $H^{s}(\mathbf{R}) \cap L^{\infty}(\mathbf{R})$ suffices to ensure uniform pointwise boundedness of the approximants. The answer is positive if $s$ is large enough since $H^{s} \subset L^{\infty}(\mathbf{R})$ for $s>1 / 2$. Thus, it is sufficient to take the data $v$ in some $H^{s}, s>1 / 2$, to ensure that the approximants are bounded uniformly in $H^{s}$, hence in $L^{\infty}(\mathbf{R})$. 
However, functions in $H^{s}$, for $s>1 / 2$, are continuous, and so our motivating example of a nonsmooth function, $J(x)$, is not locally in $H^{s}$ for $s>1 / 2$. It is natural to ask if less regularity will still suffice, i.e., we ask if assuming that the pointwise bounded data is in $H^{s}$ locally, for $s<1 / 2$, is a sufficient condition to ensure that the approximants are uniformly pointwise bounded. Here, $J(x)$ is locally in $H^{s} \cap L^{\infty}(\mathbf{R})$ for all $s<1 / 2$.

The first result shows that this is not so. The proof demonstrates that conditions made in the $L^{2}(\mathbf{R})$ norm and regularity norms based on the $L^{2}(\mathbf{R})$ norm have an inherent weakness in terms of forcing desired pointwise behavior of the approximants in situations in which dispersion is present.

Theorem 1.1. Let $E_{k}(D)$ be the operator of a dispersive constant-coefficient difference scheme which is $L^{2}(\mathbf{R})$ stable and satisfies the consistency condition for $u_{t}+c u_{x}=0, c \in \mathbf{R}$, and suppose $\lambda=k / h$ is constant. Then, there exists a function $v \in H^{s} \cap L^{\infty}(\mathbf{R})$ for all $0 \leq s<1 / 2$ such that

$$
\sup _{n k \leq 1}\left\|E_{k}^{n}(D) v\right\|_{L^{\infty}(\mathbf{R})}=\infty .
$$

Theorem 1.1 is a consequence of a more general theorem that is stated and proved in $\S 3$. In particular, the assumption of a constant mesh ratio is not needed, and therefore choosing a very small time step size, for example $k=$ $\mathrm{O}\left(h^{2}\right)$, does not help to force boundedness. The first part of this proof consists in showing that the uniform pointwise boundedness of the difference scheme operator implies that the operator whose symbol is $\exp \left(i b \xi^{\mu}\right)$, with $\mu, b \in \mathbf{R}$, $\mu \geq 2, \mu-1$ the accuracy of the scheme and $b \neq 0$ a fixed constant, is a bounded operator in $\operatorname{Hom}\left(H^{s} \cap L^{\infty}(\mathbf{R}), L^{\infty}(\mathbf{R})\right)$. Then, we produce a function $u \in H^{s} \cap L^{\infty}(\mathbf{R})$ such that

$$
\left\|e^{i b D^{\mu}} u\right\|_{L^{\infty}(\mathbf{R})}=\infty .
$$

Again, thanks to the uniform boundedness principle, this implies that there is some function in $H^{s} \cap L^{\infty}(\mathbf{R})$ which causes the difference scheme operator itself to be unbounded in the same fashion (the function $u$ may not do this). In $\S 5$, we construct a sequence of functions depending on the step size which does appear to do this in the limit as the step size tends to zero.

Returning to the computations made with the Crank-Nicolson scheme, the evidence suggests that dispersive schemes are uniformly pointwise bounded on the step function. Most of the authors mentioned above studied the boundedness and convergence of schemes on step data. Such analysis began at least partly because (1.1) with step data is considered to be a test problem for numerical methods for nonlinear hyperbolic equations. Apelkrans [1], Brenner and Thomée [5], and Thomée [22], using similar methods, studied difference approximations to the solution of the equation $u_{t}+\rho(x) u_{x}=0$, and proved convergence and gave bounds for the approximants everywhere except near the point of discontinuity, under the assumption that the schemes are $L^{p}(\mathbf{R})$ stable only for $p=2$ and with a constant mesh ratio $\lambda=k / h$ (see [6]). Taking a 
different approach for the same kind of schemes, Hedstrom [12,13] studied convergence of finite difference approximants of the equation $u_{t}+u_{x}=0$ and gave a precise description of their behavior. In the case of step data, under the additional assumption. that the scheme have some higher-order dissipative character (while remaining only $L^{2}$ stable), he showed that the approximants are pointwise bounded.

One conclusion that can be drawn from Hedstrom's result is that for partially dissipative schemes the assumption that the data be piecewise smooth is sufficient to ensure that the approximants are bounded in $L^{\infty}(\mathbf{R})$. We point out that this result has a wider significance because schemes which yield uniformly pointwise bounded approximants on step data, in fact, yield uniformly pointwise bounded approximants on data chosen in BV. BV is the class of functions determined by the norm

$$
\|f\|_{\mathrm{BV}}:=\|f\|_{L^{\infty}(\mathbf{R})}+\|D f\|_{\mathrm{TV}}
$$

that is, $\mathrm{BV}$ is the class of $L^{\infty}(\mathbf{R})$ functions whose derivatives are bounded signed Borel measures. More precisely, let $E_{k}(D)$ be a translation invariant, $L^{2}(\mathbf{R})$ stable operator such that

$$
\sup _{n k \leq 1}\left\|E_{k}^{n}(D) J(\cdot)\right\|_{L^{\infty}(\mathbf{R})} \leq C
$$

for some constant $C$. Then

$$
\sup _{n k \leq 1}\left\|E_{k}^{n}(D) v(\cdot)\right\|_{L^{\infty}(\mathbf{R})} \leq C\|v\|_{\mathrm{TV}}
$$

for all $v \in \mathrm{BV}$. The proof is easy, but we present it for the reader's convenience in $\S 4$.

Hedstrom's proof of the uniform pointwise boundedness of dispersive schemes with higher-order dissipation makes explicit use of the dissipative term. This leaves open the question of uniform pointwise boundedness of purely dispersive schemes like the Crank-Nicolson scheme. Our next result shows that these schemes are bounded, as well. We recall that the characteristic function of a difference scheme is the function $\mathbf{e}(\xi):=E_{k}(\xi / h)$.

Theorem 1.2. Let $E_{k}(D)$ be the difference operator of a constant-coefficient finite difference scheme which satisfies the consistency condition for (1.1) and whose symbol has the property that $\left|E_{k}(\xi)\right| \equiv 1$. Assume further that the characteristic function of $E_{k}(D)$ is independent of $h$ and that $k / h$ is constant. Then the difference scheme is uniformly pointwise bounded on the step function.

There are three points to be made. First, by Theorem 5.2.1 of Brenner et al. [6], if $E_{k}(D)$ is not a simple translation operator, then it is unstable in $L^{p}(\mathbf{R})$ for all $p \neq 2$. Second, schemes satisfying the conditions of Theorem 1.2 are therefore uniformly pointwise bounded on data in BV. We do not demonstrate that dispersive difference schemes are bounded in $\mathrm{Hom}(\mathrm{BV}, \mathrm{BV})$, and in fact they are not in general. Last, the method used to prove Theorem 1.2 gives more 
information about the difference approximants of a purely dispersive difference scheme computed on step data. As an example, our analysis indicates at what speed the oscillations collapse towards the position of the discontinuity and their limiting height as the step size tends to zero. In $\S 5$, we confirm our predictions with the observed performance of the Crank-Nicolson scheme.

A basic ingredient of the proof is the fact that the behavior of the difference scheme is determined on two regions. One region is a fixed number of mesh widths from the discontinuity, and the other range is the complement. We show that in the limit of the step size tending to zero, the range of lattice points on the order of $h^{(\mu-1) / \mu}$ from the discontinuity carries much of the important information about the difference approximant. For $\xi$ in the corresponding range in Fourier space, we construct a normal form for the symbol of the difference operator using the Malgrange Division Theorem and ideas from the analysis of singularities of maps and oscillatory integrals (see Arnol' d [2,3] as well as [7, 11, $15,17,28])$. This normal form is readily analyzed by stationary/nonstationary phase arguments like those of Stein [21] and Wainger [25].

The outline of the paper is as follows. We start in $\S 2$ with the proof of Theorem 1.2. Section 3 contains the proof of Theorem 1.1. The proof of the claim made about BV above is in $\S 4$. In $\S 5$ we present the results of the experiments mentioned above, together with some samples and discussion. Finally, the appendix contains the technical proof of the construction of the normal form.

\section{Proof of Theorem 1.2}

Theorem 1.2 is a consequence of

Theorem 2.1. Let $E_{k}(D)$ be a translation invariant, $L^{2}(\mathbf{R})$ stable finite difference operator with $\mathbf{e}(\xi):=E_{k}(\xi / h)$ satisfying

$$
\mathbf{e}(\xi) \text { is independent of } k \text {; }
$$

$\mathbf{e}(\xi)$ is periodic and analytic in a strip around the real axis;

$$
\begin{gathered}
|\mathbf{e}(\xi)| \equiv 1 ; \\
\log (\mathbf{e}(\xi))=-i \lambda \xi+\mathrm{o}(|\xi|) \quad \text { as } \xi \rightarrow 0 ; \\
\overline{\mathbf{e}(\xi)}=\mathbf{e}(-\xi)
\end{gathered}
$$

where $h:=\lambda k, \lambda$ a fixed real constant. Then,

$$
\sup _{n k \leq 1} \sup _{x}\left|P . V . \int e^{i x \xi} E_{k}^{n}(\xi) \frac{d \xi}{\xi}\right|<\infty .
$$

Proof of Theorem 1.2. It is clear that the conclusion of Theorem 1.2 is precisely (2.6). The properties $(2.1),(2.2)$ and (2.3) follow immediately from the assumption that $k / h$ is constant, the assumptions made on $E_{k}(D)$ and the fact that $E_{k}(D)$ is the operator of a finite difference scheme which implies that $\mathbf{e}(\xi)$ is a rational trigonometric function. (2.5) is true because $\mathbf{e}(\xi)$ is the ratio of two 
polynomial functions of $e^{i h \xi}$ in which the polynomials have real coefficients. (2.5) implies that $\log \mathbf{e}(\xi)$ is an odd function.

Remark. Assumption (2.2) is a natural condition to make on the symbol of a translation invariant finite difference scheme. In fact, we only need analyticity to guarantee that $\log (\mathbf{e}(\xi))$ has a finite number of zeros in one period. Otherwise, it would suffice to assume that $\mathbf{e}(\xi)$ is $C^{\infty}$.

The rest of this section is devoted to the proof of Theorem 2.1 and to discussing some of its consequences. Since the proof is trivial when $E_{k}(D)$ is a simple translation operator, we now assume that $E_{k}(D)$ is truly dispersive, i.e., not a simple translation operator. The difference scheme approximant computed from the step data is given by the oscillatory integral

$$
\text { P.V. } \int e^{i x \xi} E_{k}^{n}(\xi) \frac{d \xi}{\xi} .
$$

We show that this integral is uniformly pointwise bounded and describe its behavior for $x$ in the vicinity of the discontinuity of the true solution.

Consider first the formula

$$
J(x)=\mathrm{P} . \mathrm{V} . \int e^{i x \xi} \frac{d \xi}{\xi} .
$$

The principal value interpretation of this integral is used to make sense of the integrand for $\xi$ near zero and to yield convergence of the integral over $\mathbf{R}$. A dispersive difference scheme introduces oscillations whose frequencies increase with $n$. The question is: How do the oscillations affect the convergence of the principal-value integral?

As for (2.8), there are two natural scales (whose precise ranges are determined by the difference scheme) in (2.7), $\xi$ very large and $\xi$ close to zero, resp. the very fine behavior and the coarser behavior of the approximant in $x$. We use different techniques to deal with these two contributions. Write (2.7) as

$$
\text { P.V. } \int_{|\xi| \leq \varepsilon / h} e^{i x \xi} E_{k}^{n}(\xi) \frac{d \xi}{\xi}+\text { P.V. } \int_{|\xi| \geq \varepsilon / h} e^{i x \xi} E_{k}^{n}(\xi) \frac{d \xi}{\xi},
$$

where $\varepsilon>0$ is small and fixed. We treat each of the integrals in (2.9) separately.

Remark. In $x$ space, the second integral is the major contributor to the amplitude of the difference approximant for $x$ within $h$ of the discontinuity and the first integral is the major contributor for $x$ further away. We show below that in fact the largest oscillation of the Crank-Nicolson scheme occurs roughly at a distance of $1.95 h^{2 / 3}$ away from the discontinuity. This suggests that the first integral in (2.9) represents the difference scheme in the limit as $h \rightarrow 0$.

Rewrite (2.9) as

$$
\text { P.V. } \int_{h|\xi| \leq \varepsilon} e^{i x \xi} \mathbf{e}^{n}(h \xi) \frac{d \xi}{\xi}+\text { P.V. } \int_{h|\xi| \geq \varepsilon} e^{i x \xi} \mathbf{e}^{n}(h \xi) \frac{d \xi}{\xi},
$$

and change variables, using the fact that $\frac{1}{h}=\lambda n$, to get

$$
\text { P.V. } \int_{|\xi| \leq \varepsilon} e^{i \lambda n x \xi} \mathbf{e}^{n}(\xi) \frac{d \xi}{\xi}+\text { P.V. } \int_{|\xi| \geq \varepsilon} e^{i n \lambda x \xi} \mathbf{e}^{n}(\xi) \frac{d \xi}{\xi}
$$


We treat the second integral first. Start by considering the oscillatory integral

$$
I(w)(x):=\text { P.V. } \int_{|\xi| \geq \pi} e^{i x \xi} w(\xi) \frac{d \xi}{\xi}:=\lim _{M \rightarrow \infty} \int_{\pi \leq|\xi| \leq M \pi} e^{i x \xi} w(\xi) \frac{d \xi}{\xi},
$$

where $w(\xi)$ is locally integrable and periodic with period $2 \pi$.

Lemma 2.2. For the integral in (2.11) we have

$$
I(w)(x)=h(x) \int_{-\pi}^{+\pi} e^{i x \xi} w(\xi) d \xi-\int_{-\pi}^{+\pi} e^{i x \xi} w(\xi) f(x, \xi) d \xi,
$$

where $h(x)$ is the periodic function with period 1 whose restriction to the interval $[-1 / 2,1 / 2]$ is given by

$$
h(x)= \begin{cases}x+1 / 2, & -1 / 2 \leq x<0 \\ 0, & x=0 \\ x-1 / 2, & 0<x \leq 1 / 2\end{cases}
$$

and

$$
f(x, \xi):=\sum_{m=-\infty}^{\infty} e^{2 \pi i m x} \frac{\xi}{(\xi+2 \pi m) 2 \pi m},
$$

where the prime on the summation symbol indicates that the term with index zero is omitted. Moreover, $f(x, \xi)$ is uniformly bounded for $-\infty<x<\infty$ and $\xi \in[-\pi, \pi]$.

In particular,

$$
\sup _{x}\left|P . V \cdot \int_{|\xi| \geq \pi} e^{i x \xi} w(\xi) \frac{d \xi}{\xi}\right| \leq C \int_{-\pi}^{+\pi}|w(\xi)| d \xi,
$$

with a constant $C$ which is independent of $x$ and $w(\xi)$.

Proof of Lemma 2.2. For periodic $g$ with period $2 \pi$,

$$
\int_{\pi \leq|\xi| \leq M \pi} g(\xi) d \xi=\sum_{|m| \leq M}^{\prime} \int_{-\pi}^{+\pi} g(\xi+2 \pi m) d \xi
$$

thus the integral on the right in $(2.11)$ is equal to

$$
\left(\sum_{m \mid \leq M}^{\prime} e^{2 \pi i m \xi} \frac{1}{2 \pi m}\right) \int_{-\pi}^{+\pi} e^{i x \xi} w(\xi) d \xi-\int_{-\pi}^{+\pi} e^{i x \xi} w(\xi) f_{M}(x, \xi) d \xi
$$

with

$$
f_{M}(x, \xi):=\sum_{|m| \leq M}^{\prime} e^{2 \pi i m x} \frac{\xi}{(\xi+2 \pi m) 2 \pi m}
$$

Note that

$$
\sum_{|m| \leq M}^{\prime} e^{2 \pi i m x} \frac{1}{2 \pi m} \rightarrow h(x) \text { as } M \rightarrow \infty
$$

pointwise for every $x$, and the sums are uniformly bounded. Also, $f_{M}(x, \xi)$ converges uniformly to the continuous function $f(x, \xi)$ as $M \rightarrow \infty$. 
Now consider the characteristic function $\mathbf{e}(\xi)$ of a difference scheme satisfying the hypotheses of Theorem 1.2. Without loss of generality, we may assume that $\mathbf{e}(\xi)$ is periodic with smallest period equal to $2 \pi$. We can arrange this with a simple change of variables in $\xi$, using the unique smallest positive period of $\mathbf{e}(\xi)$, and since the estimates will be uniform in $x$, the change to the exponent of $e$ in the integral in (2.10) can be absorbed into $x$. Similarly, $\lambda$ also can be absorbed into $x$. Hence, we can apply Lemma 2.2 to find that

$$
\mid \text { P.V. } \int_{|\xi| \geq \pi} e^{i \lambda x \xi n} \mathbf{e}^{n}(\xi) \frac{d \xi}{\xi} \mid \leq C
$$

for all $x, \lambda$ and $n$. Since for fixed $\varepsilon>0$,

$$
\int_{\varepsilon \leq|\xi| \leq \pi} e^{i \lambda x \xi n} \mathbf{e}^{n}(\xi) \frac{d \xi}{\xi}
$$

is obviously uniformly bounded by $(2.3)$, it remains to consider

$$
\text { P.V. } \int_{|\xi| \leq \varepsilon} e^{i n(\lambda x \xi+\theta(\xi))} \frac{d \xi}{\xi},
$$

where $\theta(\xi)$ denotes the real analytic function which satisfies

$$
\mathbf{e}^{\prime}(\xi)=i \mathbf{e}(\xi) \theta^{\prime}(\xi), \quad \theta(0)=0 .
$$

It is easy to see that $\theta(2 \pi)=m 2 \pi$ for some integer $m$ and that $\mathbf{e}(\xi)=e^{i \theta(\xi)}$. Moreover, (2.4) and (2.5) imply that $\theta$ is an odd function and therefore $\theta(\xi)=$ $-\lambda \xi+c_{\mu} \xi^{\mu}+\cdots$, where $\mu$ is an odd integer greater than one and $c_{\mu} \neq 0 .(\mu-1$ is the accuracy of the difference scheme, which we have assumed is not a simple translation operator.)

$\lambda$ is a fixed real number, so replacing $n$ by $n \lambda$ and $\theta$ by $\theta / \lambda$ eliminates $\lambda$ from the integrand in (2.14). Our first aim is to show that this expression is bounded in magnitude uniformly in $n$ and $x$. The following lemma is the basic ingredient for what follows. It applies to a symbol satisfying the hypotheses of Theorem 2.1 with $\nu:=(\mu-1) / 2$.

Lemma 2.3. Let $F(\xi, y)=\xi y+(\theta(\xi)+\xi)$, where $y:=x-1$ and $(\theta(\xi)+\xi)=$ $\mathrm{O}\left(\xi^{2 \nu+1}\right)$ as $|\xi| \rightarrow 0, \nu \geq 1$. There is an open neighborhood $O$ of the origin, $B_{j} \in C^{\infty}(O)$ and $\varepsilon>0$ such that $P(\xi, y):=\xi^{2 \nu+1}+\sum_{j=0}^{\nu-1} B_{j}(y) \xi^{2 j+1}$ satisfies

$$
\sup _{y \in O}\left|P . V . \int_{|\xi| \leq \varepsilon} e^{i n F(\xi, y)} \frac{d \xi}{\xi}-P . V . \int_{|\xi| \leq \varepsilon^{\prime}} e^{i n P(\xi, y)} \frac{d \xi}{\xi}\right| \leq C
$$

for some $\varepsilon^{\prime}>0$.

Remark. $P(\xi, y)$ is not uniquely determined.

Proof of Lemma 2.3. By Theorem A1 (see the appendix), for $\xi$ and $y$ sufficiently small, there exist smooth functions $B_{j}(y), B_{j}(y)=\mathrm{O}(y)$ as $y \rightarrow 0$, 
$j=0, \ldots, \nu-1$, and $\Xi(\xi, y)$ odd in $\xi$, such that

$$
F(\xi, y)=\Xi^{2 \nu+1}+\sum_{j=0}^{\nu-1} B_{j}(y) \Xi^{2 j+1}=: P(\Xi, y) \text {. }
$$

Hence,

$$
\begin{aligned}
\text { P.V. } \int_{|\xi| \leq \varepsilon} e^{i n P(\Xi(\xi, y), y)} \frac{d \xi}{\xi}= & \text { P.V. } \int_{|\xi| \leq \varepsilon} e^{i n P(\Xi(\xi, y), y)}\left(\frac{1}{\xi}-\frac{\Xi^{\prime}}{\Xi}\right) d \xi \\
& + \text { P.V. } \int_{|\xi| \leq|\Xi(\varepsilon, y)|} e^{i n P(\xi, y)} \frac{d \xi}{\xi},
\end{aligned}
$$

where $\Xi^{\prime}=\partial \Xi / \partial \xi$, and the oddness of $\Xi$ was used. Hence, it suffices to show that

$$
\frac{1}{\xi}-\frac{\Xi^{\prime}}{\Xi}
$$

is bounded uniformly for $\xi$ and $y$ sufficiently small. Since $\Xi(-\xi, y)=$ $-\Xi(\xi, y)$,

$$
\Xi(\xi, y)-\xi \Xi^{\prime}(\xi, y)=\mathrm{O}\left(\xi^{3}\right)
$$

as $\xi \rightarrow 0$, and by (A-2) in the appendix,

$$
\frac{\Xi(\xi, y)}{\xi}=\mathrm{O}(1)
$$

as $\xi \rightarrow 0$. Therefore, $(2.17)$ is $\mathrm{O}(|\xi|)$ as $\xi \rightarrow 0$, and $(2.15)$ holds with $\varepsilon^{\prime}=$ $\Xi(\varepsilon, y)$.

Proof of Theorem 2.1. First consider the case when $x$ is taken in a small, fixed neighborhood around one. By Lemma 2.3 and the estimates made on the second integral in (2.10), the uniform pointwise boundedness of $(2.7)$ for such $x$ is reduced to the boundedness of

$$
\text { P.V. } \int_{-l}^{l} e^{i n\left(B_{0} \xi+B_{1} \xi^{3}+\cdots+\xi^{2 \nu+1}\right)} \frac{d \xi}{\xi}
$$

for some $l$. Since the coefficients $B_{0}, \ldots, B_{\nu-1}$ depend on $y$ in an uncontrollable fashion, it is crucial to get a bound on (2.19) which depends on $\nu$ but not on $B_{0}, \ldots, B_{\nu-1}$. Such a result was obtained by Stein and Wainger. The proof, from [21] and [25], is repeated here for the sake of completeness.

By the definition of P.V., the result follows if the integral

$$
\left|\int_{\varepsilon \leq|\xi| \leq 1} e^{i\left(c_{0} \xi+c_{1} \xi^{3}+\cdots+c_{\nu} \xi^{2 \nu+1}\right)} \frac{d \xi}{\xi}\right|
$$

is shown to be bounded uniformly in $c_{0}, \ldots, c_{\nu}, l$ and $\varepsilon$. Using the scale invariance of $d \xi / \xi$, we may assume that $c_{\nu}= \pm 1$. By partial integration,

$$
\int_{1}^{l / \varepsilon} e^{i\left(c_{0} \xi+\cdots \pm \xi^{2 \nu+1}\right)} \frac{d \xi}{\xi}=\left.I(\xi) \frac{1}{\xi}\right|_{1} ^{l / \varepsilon}+\int_{1}^{l / \varepsilon} I(\xi) \frac{d \xi}{\xi^{2}}
$$


where

$$
I(\xi):=\int_{1}^{l / \varepsilon} e^{i\left(c_{0} \xi+\cdots \pm \xi^{2 \nu+1}\right)} d \xi
$$

An application of Van der Corput's lemma (stated below) with $j=2 \nu+1$ shows that $I(\xi)$ is bounded in magnitude by a constant $C(\nu)$, and therefore so is the integral on the left-hand side of (2.20) independently of $l / \varepsilon$.

On the other hand,

$$
\begin{aligned}
& \left|\int_{\varepsilon \leq|\xi| \leq 1} e^{i\left(c_{0} \xi+\cdots+c_{\nu-1} \xi^{2 \nu-1} \pm \xi^{2 \nu+1}\right)} \frac{d \xi}{\xi}-\int_{\varepsilon \leq|\xi| \leq 1} e^{i\left(c_{0} \xi+\cdots+c_{\nu-1} \xi^{2 \nu-1}\right)} \frac{d \xi}{\xi}\right| \\
& \quad \leq \int_{\varepsilon \leq|\xi| \leq 1} \xi^{2 \nu+1} \frac{d \xi}{\xi} \leq C(\nu),
\end{aligned}
$$

independent of $\varepsilon$. Thus, by using induction, the problem is reduced to the case when $2 \nu+1=1$, which is a standard result.

Van der Corput's Lemma. Let $\psi \in C^{j}\left(\mathbf{R}^{1}\right)$ be real with $\left|\psi^{(j)}(\xi)\right| \geq \delta>0$ on $[a, b]$. Then

$$
\left|\int_{a}^{b} e^{i \psi} d \xi\right| \leq C
$$

with $C$ independent of $a$ and $b$.

This lemma is proved using the same lemmas of Stein that were used above. Stein proves the following related proposition in [21].

"Proposition 2". Suppose that $\phi$ is real-valued and smooth in $[a, b]$. If $\left|\phi^{(j)}(x)\right|$ $\geq 1$, then

$$
\left|\int_{a}^{b} e^{i n \phi(x)} d x\right| \leq c_{j} n^{-1 / j}
$$

holds when (i) $j \geq 2$ or (ii) $j=1$, if in addition it is assumed that $\phi^{\prime}(x)$ is monotonic.

"Corollary". Under these assumptions on $\phi$, we can conclude that

$$
\left|\int_{a}^{b} e^{i n \phi(x)} \psi(x) d x\right| \leq c_{j} n^{-1 / j}\left\{|\psi(b)|+\int_{a}^{b}\left|\psi^{\prime}(x)\right| d x\right\},
$$

where $\psi$ is any smooth complex-valued function.

Remark. That (2.14) is bounded uniformly for $x$ outside of a small neighborhood of one is easier to show. In this case, $(\lambda x \xi+\theta(\xi))^{\prime}=\lambda(x-1)+o(|\xi|)$ is uniformly bounded away from zero for $\xi$ in a small neighborhood of zero. Therefore, one can construct a map $\bar{\Xi}(\xi, x-1)$ with

$$
x \lambda \bar{\Xi}(\xi, x-1)+\theta(\bar{\Xi}(\xi, x-1))=(x-1) \lambda \xi+B \xi^{2 \nu+1},
$$

for some real constant $B$, by the implicit function theorem. Then, one proceeds as above. Of course, it is necessary for $|x-1|$ to be bounded away from zero, 
the boundedness of the integral with the transformed variable will depend on $|x-1|^{-1}$.

Next we prove

Theorem 2.4. For $x$ sufficiently close to 1 ,

$$
\lim _{n \rightarrow \infty}\left|P . V . \int_{-\infty}^{\infty} e^{i \lambda n \xi x} \mathbf{e}^{n}(\xi) \frac{d \xi}{\xi}-P . V . \int_{-\varepsilon^{\prime}}^{\varepsilon^{\prime}} e^{i n P(\xi, x-1)} \frac{d \xi}{\xi}\right|=0 .
$$

In other words, in the vicinity of the discontinuity of the true solution, the behavior of the difference scheme is determined by the normal form $P(\xi, x-1)$.

Proof of Theorem 2.4. Thanks to Lemma 2.3, it suffices to show that the second integral in (2.10) and the first integral on the right of (2.16) tend to zero as $n \rightarrow \infty$, uniformly in $\lambda x$. Consider the former integral. Once again, we let $x$ replace $\lambda x$ and assume that $\mathbf{e}(\xi)$ is periodic with smallest period $2 \pi$; thus we consider

$$
\text { P.V. } \int_{|\xi| \geq \pi} e^{i x \xi n} \mathbf{e}^{n}(\xi) \frac{d \xi}{\xi} .
$$

Returning to (2.12), we analyze this expression with $w(\xi)=\mathbf{e}^{n}(\xi)$, using stationary and nonstationary phase methods. The stationary phase points of $e^{i x \xi} \mathbf{e}(\xi)$ are given by the zeros of the function

$$
i x+\frac{\mathbf{e}^{\prime}(\xi)}{\mathbf{e}(\xi)}=i x+i \theta^{\prime}(\xi) \text {. }
$$

It follows from the assumptions made on $\mathbf{e}(\xi)$ that $\theta^{\prime \prime}(\xi)$ is not identically zero on any subinterval of $[-\pi, \pi]$, and because $\theta$ is analytic in a strip around the real axis, $\theta^{\prime \prime}(\xi)$ has only a finite number of zeros, $M$, in $[-\pi, \pi]$. Call these zeros $\xi_{1}, \ldots, \xi_{M}$ with multiplicities $\mu_{1}-1, \ldots, \mu_{M}-1$. Choose $\delta$ positive so that $\theta^{\prime \prime}(\xi)$ has only one zero in each neighborhood $N_{j}=\left\{\xi|| \xi-\xi_{j} \mid<\delta\right\}$ of $\xi_{j}, j=1, \ldots, M$, and furthermore, so that there exists a constant $c=c(\delta)$ such that $\left|\theta^{\left(\mu_{j}\right)}(\xi)\right| \geq c$ for $\xi \in N_{j}, j=1, \ldots, M$, and $\left|\theta^{\prime \prime}(\xi)\right| \geq c$ for $\xi \in[-\pi, \pi] \backslash \bigcup N_{j}$. Since $\left(\partial^{j} / \partial \xi^{j}\right)(x \xi+\theta(\xi))=\theta^{(j)}, j \geq 2$, these bounds also hold for $x \xi+\theta(\xi)$, independent of $x$.

In view of (2.12) and (2.13), consider two terms. First,

$$
\begin{aligned}
\int_{-\pi}^{+\pi} & e^{i n(x \xi+\theta(\xi))} f(x n, \xi) d \xi \\
& =\int_{-\pi}^{+\pi} e^{i n(x \xi+\theta(\xi))} \sum^{\prime} e^{2 \pi i m x} \frac{\xi}{(\xi+2 \pi m) 2 \pi m} d \xi .
\end{aligned}
$$

Using Fubini's theorem, we can switch the order of summation and integration.

Thus, it suffices to treat

$$
e^{2 \pi i m x} \int_{-\pi}^{+\pi} e^{i n(x \xi+\theta(\xi))} \frac{\xi}{(\xi+2 \pi m) 2 \pi m} d \xi, \quad m \neq 0,
$$


separately and then sum the results. We decompose this integral as

$$
\begin{aligned}
& \sum_{j=1}^{M} \int_{N_{j}} e^{i n(x \xi+\theta(\xi))} \frac{\xi}{(\xi+2 \pi m) 2 \pi m} d \xi \\
& \quad+\int_{[-\pi, \pi] \backslash \cup N_{j}} e^{i n(x \xi+\theta(\xi))} \frac{\xi}{(\xi+2 \pi m) 2 \pi m} d \xi .
\end{aligned}
$$

Since $\theta^{\prime \prime}$ is of constant sign on each component of $[-\pi, \pi] \backslash \bigcup N_{j}$, we can use the corollary to Proposition 2 of Stein [21] to conclude that the second integral is bounded in magnitude by

$$
c n^{-1 / 2} \frac{1}{m^{2}} .
$$

By the same corollary, each integral in the sum in (2.22) is bounded in magnitude by

$$
c n^{-1 /(\mu,+1)} \frac{1}{m^{2}} .
$$

Summing (2.23) and (2.24) in $m$, we see that the integral in (2.21) can be made as small as we like by choosing $n$ large.

The other term in (2.12) is

$$
h(x) \int_{-\pi}^{+\pi} e^{i x \xi n} \mathbf{e}^{n}(\xi) d \xi .
$$

This can be handled in the same fashion as were the integrals in the sum in (2.21), now using Proposition 2 of Stein [21] directly, yielding the same asymptotic bounds.

Using arguments similar to those used to bound the integrals in (2.22), it is easy to see that the contributions to the second integral in (2.10),

$$
\int_{\varepsilon \leq|\xi| \leq \pi} e^{i \lambda x \xi n} \mathbf{e}^{n}(\xi) \frac{d \xi}{\xi},
$$

also tend to 0 as $n \rightarrow \infty$ at the same asymptotic rate uniformly in $x$ and $\lambda$ for any fixed $\varepsilon>0$. Hence, we conclude that in the limit of the step size tending to zero, the difference scheme solution is described by the first integral in $(2.10)$ with an error like $\mathrm{O}\left(n^{-1 /(\bar{\mu}+1)}\right), \bar{\mu}=\max \mu_{i}$.

Now, we look at the first term on the right of $(2.16)$,

$$
\int_{-\varepsilon}^{\varepsilon} e^{i n F(\xi, y)}\left(\frac{1}{\xi}-\frac{\Xi^{\prime}}{\Xi}\right) d \xi,
$$

where $1 / \xi-\Xi^{\prime} / \Xi$ is a smooth function of $\xi$ and $y$. Arguments similar to those above show that this term tends to zero as $n \rightarrow \infty$ uniformly for $x$ in a neighborhood of 1 .

The normal form can be used now to analyze some of the oscillations appearing in the difference approximant around the discontinuity of the solution. 
Since for fixed $y \neq 0$ the scheme converges to the actual solution, one might be tempted to ask how quickly the peaks of the oscillations move towards the discontinuity and how large these peaks can be. In other words, how to choose $x(n), x(n) \rightarrow 1$ as $n \rightarrow \infty$, such that the expression (2.14) deviates substantially from the true solution of the differential equation. The following result shows that for difference schemes with normal form $P$ the approximate solution $U\left(1,1+a h^{2 \nu /(2 \nu+1)}\right)$ is roughly independent of $h$ as $h \rightarrow 0$. This suggests the rate at which peaks collapse towards the discontinuity and gives a formula which can be used to estimate the heights of the peaks (see $\S 5 \mathrm{~B}$ ).

Theorem 2.5. Let $y(n)=a n^{-2 \nu /(2 \nu+1)}$. Then,

$$
\lim _{n \rightarrow \infty} P . V . \int_{-\varepsilon^{\prime}}^{\varepsilon^{\prime}} e^{i n P(\xi, y(n))} \frac{d \xi}{\xi}=P . V . \int_{-\infty}^{\infty} e^{i\left(c a \xi+\xi^{2 \nu+1}\right)} \frac{d \xi}{\xi},
$$

where

$$
c:=\frac{d B_{0}}{d y}(0)=\left(\frac{\partial \Xi}{\partial \xi}(0,0)\right)^{-1} \neq 0 .
$$

Proof. Since $B_{j}(y)=\mathrm{O}(y), j=0, \ldots, \nu-1$, changing variables $\xi \rightarrow$ $n^{-1 /(2 \nu+1)} \xi$,

$$
\begin{aligned}
Q_{n}(\xi, a):=n P\left(n^{-1 /(2 \nu+1)} \xi, y(n)\right) \\
=\xi^{2 \nu+1}+\sum_{j=0}^{\nu-1} B_{j}(y(n)) n^{1-(2 j+1) /(2 \nu+1)} \xi^{2 j+1} \\
=\xi^{2 \nu+1}+B_{0}\left(n^{-2 \nu /(2 \nu+1)} a\right) n^{2 \nu /(2 \nu+1)} \xi+o(1),
\end{aligned}
$$

which establishes the result provided the interchange of the limit with the principle value integral is justified. Obviously, the zeros of (2.26) for $a$ fixed, all stay bounded inside a circle of radius say $R$, uniformly in $n$. Now

$$
\lim _{n \rightarrow \infty}\left(\text { P.V. } \int_{-R}^{R} e^{i Q_{n}(\xi, a)} \frac{d \xi}{\xi}-\text { P.V. } \int_{-R}^{R} e^{i\left(c a \xi+\xi^{2 a+1}\right)} \frac{d \xi}{\xi}\right)=0
$$

by the dominated convergence theorem, observing that $S_{n}(\xi):=e^{i Q_{n}(\xi, a)}-$ $e^{i\left(c a \xi+\xi^{2 \nu+1}\right)}=\mathrm{O}(\xi)$ uniformly in $n$ and $S_{n}(\xi) \rightarrow 0$ pointwise as $n \rightarrow \infty$.

To treat

rewrite it as

$$
\text { P.V. } \int_{|\xi| \geq R} e^{i Q_{n}(\xi, a)} \frac{d \xi}{\xi},
$$

$$
\begin{aligned}
\text { P.V. } & \int_{|\xi| \geq R} e^{i Q_{n}(\xi, a)}\left(\frac{1}{\xi}-\frac{1}{2 \nu+1} \frac{Q_{n}^{\prime}(\xi, a)}{Q_{n}(\xi, a)}\right) d \xi \\
& + \text { P.V. } \int_{|\xi| \geq R} e^{i Q_{n}(\xi, a)} \frac{1}{2 \nu+1} \frac{Q_{n}^{\prime}(\xi, a)}{Q_{n}(\xi, a)} d \xi,
\end{aligned}
$$

which is allowed since all of the zeros of $Q_{n}(\xi, a)$ lie inside $\{\xi|| \xi \mid \leq R\}$. 
To interchange limit and integral in (2.27), observe that

$$
\left|\frac{Q_{n}(\xi, a)-\frac{1}{2 \nu+1} Q_{n}^{\prime}(\xi, a) \xi}{\xi Q_{n}(\xi, a)}\right| \leq \frac{c}{|\xi|^{3}}
$$

for some fixed constant $C$ and that the integrand converges pointwise to

$$
\left(1-\frac{1}{2 \nu+1}\right) e^{i\left(c a \xi+\xi^{2 \nu+1}\right)} \frac{c a}{\xi^{2 \nu+1}+c a \xi},
$$

and use dominated convergence. The change of variables $\xi=Q_{n}(\xi, a)$ in the second integral of (2.27) yields

$$
\frac{1}{2 \nu+1} \text { P.V. } \int_{|\xi| \geq\left|Q_{n}(R, a)\right|} e^{i \xi} \frac{d \xi}{\xi}
$$

which converges to

$$
\begin{aligned}
& \frac{1}{2 \nu+1} \text { P.V. } \int_{|\xi| \geq\left|R^{2 \nu+1}+c a R\right|} e^{i \xi} \frac{d \xi}{\xi} \\
& \quad=\frac{1}{2 \nu+1} \text { P.V. } \int_{|\xi| \geq R} e^{i\left(\xi^{2 \nu+1}+c a \xi\right)} \frac{(2 \nu+1) \xi^{2 \nu}+c a}{\xi^{2 \nu+1}+c a \xi} d \xi .
\end{aligned}
$$

Adding (2.28) and (2.29) yields the result.

\section{Proof of Theorem 1.1}

Theorem 1.1 follows once we have proved Theorem 3.1 given below. This theorem is stated in terms of the Besov spaces $\mathrm{B}_{2}^{s, q}$ rather than the $H^{s}$ spaces because the Besov spaces are finer.

First, we recall the definition of the Besov spaces.

Definition: Besov spaces $\mathrm{B}_{2}^{s, q}, 1 \leq q \leq \infty, 0<s$. Let $\left\{\psi_{j}(\xi), j \geq 0\right\}$ be a set of nonnegative functions with

$$
\psi_{0} \in C_{0}^{\infty}(\{\xi:|\xi|<2\}), \quad \psi_{j} \in C_{0}^{\infty}\left(\left\{\xi: 2^{j-1}<|\xi|<2^{j+1}\right\}\right),
$$

and $\sum \psi_{j}(\xi) \equiv 1$. Then, $v \in L^{2}(\mathbf{R})$ is in $\mathrm{B}_{2}^{s, q}$ if and only if

$$
\|v\|_{\mathbf{B}_{2}^{s, q}}:=\left\|\left\{2^{j s}\left\|\mathscr{F}^{-1}\left(\psi_{j} \hat{v}\right)\right\|_{L^{2}(\mathbf{R})}\right\}\right\|_{l_{q}\left(\mathbf{Z}^{+}\right)}<\infty,
$$

where $l_{q}\left(\mathbf{Z}^{+}\right)$is the space of sequences $\left\{\left\{c_{j}\right\}, j \in \mathbf{Z}, j \geq 0\right\}$ with the usual norm.

We note that $\mathscr{F}^{-1}\left(\psi_{j} \hat{v}\right)$ is the "part" of $v$ with frequency close to $\pm 2^{j}$, and that the Besov norm measures the growth of these parts as $j \rightarrow \infty$.

Example 3.1. The integral $J(x)$ in (2.8) is locally in $\mathrm{B}_{2}^{1 / 2, q}$ if and only if $q=\infty$.

We call these spaces finer because the following inclusions hold:

$$
\bigcup_{s>1 / 2} H^{s} \subset \mathrm{B}_{2}^{1 / 2,1} \subset \mathrm{B}_{2}^{1 / 2, q} \subset \mathrm{B}_{2}^{1 / 2, \infty} \subset \bigcap_{s<1 / 2} H^{s}
$$


for all $q \in[1, \infty)$. We note that $H^{s}=\mathrm{B}_{2}^{s, 2}$. By interpolation, it follows that $L^{2}(\mathbf{R})$ stable difference schemes will be stable in the spaces $\mathrm{B}_{2}^{1 / 2, q}$ as well. The sharp Sobolev theorem (see [15]) asserts that functions in $\mathbf{B}_{2}^{1 / 2,1}$ are continuous and pointwise bounded. Taking our data in this space suffices to ensure that the approximants are uniformly bounded in this space, hence in $L^{\infty}(\mathbf{R})$, and it is natural to ask whether taking the data in $B_{2}^{1 / 2, \infty} \cap L^{\infty}(\mathbf{R})$ is sufficient to ensure uniform pointwise boundedness of the approximants. $\mathrm{B}_{2}^{1 / 2, \infty} \cap L^{\infty}(\mathbf{R})$ is a small Banach space that allows step discontinuities and is contained in $L^{\infty}(\mathbf{R})$.

Recall that along with an assumption of $L^{2}(\mathbf{R})$ stability in the difference schemes, the previous works cited have in common the assumption of a constant mesh ratio. However, there are schemes for which it is efficient or even necessary to assume a different relationship between $k$ and $h$. For example, in the LaxWendroff scheme, we could take $k=h^{2}$, and satisfy the CFL condition. One might ask if sacrificing efficiency in this way will result in a scheme which is $L^{\infty}(\mathbf{R})$ stable. This is not answered in the existing literature. (See $\S 5$ for more examples.) We state the theorem in a general fashion to widen its applicability to schemes like these. In particular, we do not assume that there is a constant mesh ratio. We let $\mathscr{B}$ denote the space $\mathrm{B}_{2}^{1 / 2, \infty} \cap L^{\infty}(\mathbf{R})$.

Theorem 3.1. Let $\left\{E_{k}(D), 0<k<1\right\}$ be a family of translation invariant, $L^{2}(\mathbf{R})$ stable operators. Assume that the symbols $\left\{E_{k}(\xi)\right\}$ belong to $C^{\infty}(\mathbf{R})$ and further that there exists a $\xi_{0}$ such that

$$
\begin{gathered}
\left|E_{k}\left(\xi_{0}\right)\right| \equiv 1, \\
E_{k}\left(\xi_{0}+\xi\right)=E_{k}\left(\xi_{0}\right) \exp \left\{i a_{0} k \xi+i b_{0} k^{1+\gamma} \xi^{\mu}+k \rho_{0}(k, \xi)\right\},
\end{gathered}
$$

where $a_{0}, b_{0}, \gamma$ and $\mu$ are real, $\gamma>0$ and $\mu \geq 2 ; \rho_{0}$ is a continuous function with $\rho_{0}(0, \xi)=\rho_{0}(k, 0)=0$ and

$$
\lim _{k \rightarrow 0} \rho_{0}\left(k, k^{-\gamma / \mu} \xi\right)=0,
$$

uniformly for $\xi$ in compact sets.

Then, there exists a $u \in \mathscr{B}$ such that

$$
\sup _{n k \leq 1}\left\|E_{k}^{n}(D) u\right\|_{L^{\infty}(\mathbf{R})}=\infty .
$$

Remark. Thus, the family of operators $\left\{E_{k}(D), n k \leq 1\right\}$ is unbounded in $\operatorname{Hom}\left(\mathscr{B}, L^{\infty}\right)$ and therefore in $\operatorname{Hom}\left(H^{s} \cap L^{\infty}, L^{\infty}\right)$ for $s \in[0,1 / 2)$.

Example 3.2. For the Lax-Wendroff scheme with $k=h^{2}$, the expansion at $\xi=0$ is

$$
E_{k}(\xi)=\exp \left\{-i \xi k+i \xi^{3} k^{2}+k \rho_{0}(k, \xi)\right\},
$$

where $\rho_{0}(k, \xi)$ satisfies the hypothesis of the theorem.

The proof of Theorem 3.1 is by contradiction. The following theorem is the first step. 
Theorem 3.2. Let $\left\{E_{k}(D), 0<k<1\right\}$ be the family of operators from the statement of Theorem 3.1. If there exists a $C^{\prime}>0$ such that

$$
\left\|E_{k}^{n}(D)\right\|_{\mathscr{B} \rightarrow L^{\infty}(\mathbf{R})} \leq C^{\prime} \quad \text { for all } n k \leq 1, k \in(0,1),
$$

then $\exp \left\{i b_{0} n k D^{\mu}\right\} \in \operatorname{Hom}\left(\mathscr{B}, L^{\infty}\right)$ for all $n k \leq 1, k \in(0,1)$, and further, there is a $C=C\left(\xi_{0}\right)>0$ with

$$
\left\|\exp \left\{i b_{0} n k D^{\mu}\right\}\right\|_{\mathscr{B} \rightarrow L^{\infty}(\mathbf{R})} \leq C \text { for all } n k \leq 1, k \in(0,1) .
$$

Assuming Theorem 3.2, we conclude that if the family of operators from the statement of Theorem 3.1 is uniformly bounded in $\operatorname{Hom}\left(\mathscr{B}, L^{\infty}\right)$, then the family of operators $\left\{\exp \left\{i b_{0} t D^{\mu}\right\}, t=n k \leq 1,0<k<1\right\}$, where $b_{0} \in \mathbf{R} \backslash\{0\}$ is constant, is uniformly pointwise bounded on data in $\mathscr{B}$. Theorem 3.2 is a partial analogue of Theorem 5.2.1 of Brenner, Thomée and Wahlbin (see [6, Chapter 5]). In particular, fix $n, t=n k$, and set $b:=b_{0} t$; then the theorem implies that the operator $\exp \left\{i b D^{\mu}\right\}$ is in $\operatorname{Hom}\left(\mathscr{B}, L^{\infty}\right)$. After the proof of Theorem 3.2, we show that the function $u$,

$$
u(x):=\mathscr{F}^{-1}\left(e^{-i b \xi^{p}} \frac{\kappa(\xi)}{\xi}\right),
$$

where $\kappa(\xi) \in C^{\infty}, 0 \leq \kappa \leq 1, \kappa \equiv 0$ for $\xi \leq 1$ and $\kappa \equiv 1$ for $\xi \geq 2$, is in $\mathscr{B}$ and is mapped by $\exp \left\{i b D^{\mu}\right\}$ into $\mathscr{F}^{-1}(\kappa(\xi) / \xi) \notin L^{\infty}(\mathbf{R})$, thereby proving Theorem 3.1.

Proof of Theorem 3.2. By the assumed expansion of $E_{k}(\xi)$ at $\xi_{0}$, we have

$$
E_{k}^{n}\left(\xi_{0}+\xi\right)=E_{k}^{n}\left(\xi_{0}\right) \exp \left\{i a_{0} t \xi+i b_{0} t k^{\gamma} \xi^{\mu}+t \rho_{0}(k, \xi)\right\},
$$

where $t=n k \leq 1,0<k<1$. We define

$$
\bar{E}_{k, t}(\xi):=\left(E_{k}^{n}\left(\xi_{0}\right)\right)^{-1} \exp \left\{-i a_{0} t \xi\right\} E_{k}^{n}\left(\xi_{0}+\xi\right) .
$$

Using some simple estimates, the boundedness assumption on $E_{k}$ and the following lemma with $l(x, \xi)=\exp (i x \xi)$, we conclude that

$$
\left\|\bar{E}_{k, t}(D)\right\|_{\mathscr{B} \rightarrow L^{\infty}(\mathbf{R})} \leq C
$$

for all $k \in(0,1), t=n k \leq 1$, where $C=C\left(\left|\xi_{0}\right|\right)$ is some constant.

Lemma 3.3. Suppose the function $l(x, \xi)$ has a continuous derivative in $x$ and is continuous in $\xi$, and further, for each $\xi \in \mathbf{R}$, there is a $C^{\prime}=C^{\prime}(\xi)$ such that

$$
\max \left\{\|l(x, \xi)\|_{L^{\infty}\left(\mathbf{R}_{x}\right)},\left\|\partial_{x} l(x, \xi)\right\|_{L^{\infty}\left(\mathbf{R}_{x}\right)}\right\} \leq C^{\prime} .
$$

Then, $g(x) \in \mathscr{B}$ implies $l(x, \xi) g(x) \in \mathscr{B}$ for each $\xi \in \mathbf{R}$, and further, there is a $C=C(\xi)$ such that

$$
\|l(x, \xi) g(x)\|_{\mathscr{B}} \leq C\|g(x)\|_{\mathscr{B}} .
$$


In addition, $C$ may be chosen to be a monotonically increasing function of $|\xi|$, so that $C$ is uniformly bounded on bounded subsets of $\mathbf{R}$.

This lemma follows immediately from Theorem 2.1 of Peetre and Thomée [18]. We now set

$$
\overline{\bar{E}}_{k, t}(\xi):=\bar{E}_{k, t}\left(k^{-\gamma / \mu} \xi\right)
$$

and use

Lemma 3.4. If $A(D) \in \operatorname{Hom}\left(\mathscr{B}, L^{\infty}(\mathbf{R})\right)$, then for any constant $c>1$,

$$
\|A(c D)\|_{\mathscr{B} \rightarrow L^{\infty}(\mathbf{R})} \leq\|A(D)\|_{\mathscr{B} \rightarrow L^{\infty}(\mathbf{R})} .
$$

This lemma implies that

$$
\left\|\overline{\bar{E}}_{k, t}(D)\right\|_{\mathscr{B} \rightarrow L^{\infty}(\mathbf{R})} \leq C \quad \text { for every } k \in(0,1), n k=t \leq 1 .
$$

The proof of Lemma 3.4 is postponed for the moment. We need two other facts, as well. First, note that by the assumed $L^{2}(\mathbf{R})$ stability of $E_{k}$,

$$
\left|\overline{\bar{E}}_{k, t}(\xi)\right| \leq C \text { for every } \xi \in \mathbf{R},
$$

for some $C$. Next,

$$
\mathscr{E}(n k, \xi)=\mathscr{E}(t, \xi):=\exp \left\{i b_{0} t \xi^{\mu}\right\}=\lim _{\substack{k \rightarrow 0 \\ n k=t}} \overline{\bar{E}}_{k, t}(\xi),
$$

uniformly for $x$ in compact sets. By taking the Fourier transform of both sides and using (3.2) and (3.3), one can show that

$$
\mathscr{E}(t, D) v=S_{\substack{n \rightarrow \infty \\ n k=t}}^{\prime} \lim _{\substack{\infty \\ \text { ent }}} \overline{\bar{E}}_{k, t}(D) v \text { for } v \in S .
$$

Therefore, for $\psi \in S$, with $\int|\psi|=1$, using (3.1), we have

$$
|\langle\mathscr{E}(t, D) v, \psi\rangle| \leq C\|v\|_{\mathscr{B}},
$$

so by the converse to Hölder's inequality, $\mathscr{E}(t, D) v \in L^{\infty}(\mathbf{R})$ and

$$
\|\mathscr{E}(t, D) v\|_{L^{\infty}(\mathbf{R})} \leq C\|v\|_{\mathscr{B}}
$$

for all $v \in S$ and $t=n k \leq 1, k \in(0,1)$.

However, $S$ is not dense in $\mathscr{B}$ (see [6, p. 44]), so the usual completion argument does not suffice to prove the theorem. Nevertheless, $\mathscr{B}$ is the dual of a Banach space, namely,

$$
\mathscr{B}=\left(\mathbf{B}_{2}^{1 / 2,1} \cup L^{1}(\mathbf{R})\right)^{\prime} .
$$

Furthermore, $S$ is weak star dense in $\mathscr{B}$. In fact, it is not hard to show that if $j(x) \in C_{0}^{\infty}(-1,1), j(0)=1, j \geq 0$, and $\int j d x=1$, then for $u \in \mathscr{B}$, $u_{n}:=(n j(x n)) *(j(x / n) u(x))$ converges weak star to $u$ as $n \rightarrow \infty$, and

$$
\|u\|_{\mathscr{B}}=\lim _{n \rightarrow \infty}\left\|u_{n}\right\|_{\mathscr{B}} .
$$


Given $u \in \mathscr{B}$, we estimate

$$
\|\mathscr{E}(t, D) u\|_{L^{\infty}(\mathbf{R})}
$$

by choosing $u_{n} \in \mathscr{B}$, as above. Then $\mathscr{E}(t, D) u_{n} \rightarrow \mathscr{E}(t, D) u$ in $S^{\prime}$, so that

$$
\|\mathscr{E}(t, D) u\|_{L^{\infty}(\mathbf{R})} \leq \varlimsup_{n}\left\|\mathscr{E}(t, D) u_{n}\right\|_{L^{\infty}(\mathbf{R})} \leq C \varlimsup_{n}\left\|u_{n}\right\|_{\mathscr{B}}
$$

using (3.4). Since the right-hand side equals $C\|u\|_{\mathscr{B}}$, the proof of Theorem 3.2 is complete.

Proof of Lemma 3.4. We set $\zeta=c \xi$ and compute to find that for $u \in \mathscr{B}$,

$$
\begin{aligned}
& \|A(c D) u\|_{L^{\infty}(\mathbf{R})} \\
& \quad \leq c^{-1}\|A(D)\|_{\mathscr{B} \rightarrow L^{\infty}(\mathbf{R})}\left(\left\|\mathscr{F}^{-1} \hat{u}\left(c^{-1} \cdot\right)\right\|_{\mathrm{B}_{2}^{1 / 2, \infty}}+\left\|\mathscr{F}^{-1} \hat{u}\left(c^{-1} \cdot\right)\right\|_{L^{\infty}(\mathbf{R})}\right) .
\end{aligned}
$$

We first evaluate the Besov norm on the right, by using an (equivalent) definition (see [6]):

$$
\begin{aligned}
\| \mathscr{F}^{-1} & \hat{u}\left(c^{-1} \cdot\right) \|_{\mathrm{B}_{2}^{1 / 2}, \infty} \\
= & \left\|\mathscr{F}^{-1} \hat{u}\left(c^{-1} \cdot\right)\right\|_{L^{2}(\mathbf{R})} \\
& +\sup _{t>0} t^{1 / 2} \sup _{|y| \leq t}\left\|\mathscr{F}^{-1} \hat{u}\left(c^{-1} \cdot\right)(x+y)-\mathscr{F}^{-1} \hat{u}\left(c^{-1} \cdot\right)(x)\right\|_{L^{2}(\mathbf{R})} .
\end{aligned}
$$

To calculate the first term on the right-hand side, use Plancherel's theorem and set $\eta=c^{-1} \xi$, to obtain

$$
\left\|\mathscr{F}^{-1} \hat{u}\left(c^{-1} \cdot\right)\right\|_{L^{2}(\mathbf{R})}=c^{1 / 2}\|u\|_{L^{2}(\mathbf{R})} .
$$

For the second term, setting $\zeta=c^{-1} \xi$, we find that

$$
\left\|\left(\tau_{y}-1\right) \mathscr{F}^{-1} \hat{u}\left(c^{-1} \cdot\right)\right\|_{L^{2}(\mathbf{R})}=c^{1 / 2}\left\|\left(e^{i(y c) \zeta}-1\right) \hat{u}(\zeta)\right\|_{L^{2}\left(\mathbf{R}_{\xi}\right)} .
$$

Thus, we get for the supremum in (3.7), using in order the substitutions $z=c y$ and $t=c^{-1} s$ and also using Plancherel's theorem,

$$
c^{1 / 2} \sup _{t>0} t^{1 / 2} \sup _{|y| \leq t}\left\|\left(e^{i(y c) \zeta}-1\right) \hat{u}(\zeta)\right\|_{L^{2}\left(\mathbf{R}_{\zeta}\right)}=\sup _{s>0} s^{1 / 2} \sup _{|y| \leq s}\|u(x+y)-u(x)\|_{L^{2}(\mathbf{R})} \text {. }
$$

Since $c \geq 1$, we conclude that

$$
\left\|\mathscr{F}^{-1} \hat{u}\left(c^{-1} \cdot\right)\right\|_{\mathrm{B}_{2}^{1 / 2, \infty}} \leq c^{1 / 2}\|u\|_{\mathrm{B}_{2}^{1 / 2, \infty}} .
$$

To complete the estimate for $\left\|\mathscr{F}^{-1} \hat{u}\left(c^{-1} \cdot\right)\right\|_{\mathscr{B}}$, we set $\zeta=c^{-1} \xi$ and estimate

$$
\left\|\mathscr{F}^{-1} \hat{u}\left(c^{-1} \cdot\right)\right\|_{L^{\infty}(\mathbf{R})}=c\|u\|_{L^{\infty}(\mathbf{R})} .
$$

Finally,

$$
\begin{aligned}
\|A(c D) u\|_{L^{\infty}(\mathbf{R})} & \leq c^{-1}\|A(D)\|_{\mathscr{B} \rightarrow L^{\infty}(\mathbf{R})}\left\{c^{1 / 2}\|u\|_{\mathrm{B}_{2}^{1 / 2, \infty}}+c\|u\|_{L^{\infty}(\mathbf{R})}\right\} \\
& \leq\|A(D)\|_{\mathscr{B} \rightarrow L^{\infty}(\mathbf{R})}\|u\|_{\mathscr{B}} .
\end{aligned}
$$

This is the desired inequality. 
Proof of Theorem 3.1. As remarked after the statement of Theorem 3.2, it suffices to show that the distribution $u \in S^{\prime}(\mathbf{R})$ whose Fourier transform is $e^{i \xi^{\mu}} \kappa(\xi) / \xi, \mu \geq 2$, satisfies $u \in \mathscr{B}$. That $u \in \mathrm{B}_{2}^{1 / 2, \infty}$ is immediate. It remains to show that $u \in L^{\infty}(\mathbf{R})$.

We interpret this distribution as a principal-value integral, finding that

$$
u(x)=\text { P.V. } \int e^{i x \xi} e^{i \xi^{\mu}} \frac{\kappa(\xi)}{\xi} d \xi=\lim _{R \rightarrow \infty} \int_{0}^{R} e^{i\left(x \xi+\xi^{\mu}\right)} \frac{\kappa(\xi)}{\xi} d \xi .
$$

We analyze the last integral by the same methods used in the previous section. Since $\kappa(\xi) / \xi$ is a smooth function, we can apply the corollary to Proposition 2 of Stein [21] with $j=\mu$ to find that

$$
\left|\int_{0}^{R} e^{i\left(x \xi+\xi^{\mu}\right)} \frac{\kappa(\xi)}{\xi} d \xi\right| \leq C\left(\frac{1}{R}+\int_{0}^{R} \frac{\kappa(\xi)}{\xi^{2}} d \xi\right),
$$

where $C$ is independent of $R$. Taking the limit as $R \rightarrow \infty$ shows the result.

\section{BOUNDEDNESS OF FUNCTIONS IN BV}

This section contains the proof of the claim concerning the step function and BV made in the introduction. Our assumptions imply, first, that $E_{k}$ is uniformly pointwise bounded on the characteristic function $\chi(x):=\chi((-\infty, x])$ and, second, that it suffices to show that the claim holds for all functions $v \in \mathrm{NBV}$ (the class of functions which are of bounded variation and normalized in the sense that they are left continuous at every point of $\mathbf{R}$ and have a limit of zero at $-\infty)$. In this case, $v \in \mathrm{NBV}$ can be written

$$
v(x)=\int_{\mathbf{R}} \chi_{x} d \mu_{v},
$$

where $\mu_{v}$ is a signed Borel measure with $\int\left|d \mu_{v}\right|=\|v\|_{\mathrm{TV}}<\infty$.

Choose $\psi \in S$ with $\int|\psi|=1$ and consider the definition of $E_{k}^{n}(D) v$ as an element in $S^{\prime}$ :

$$
\left\langle E_{k}^{n}(D) v, \psi\right\rangle=\left\langle v, E_{k}^{n}(-D) \psi\right\rangle=\left\langle\int \chi_{x} d \mu_{v}, E_{k}^{n}(-D) \psi(x)\right\rangle .
$$

Using Fubini's theorem to switch the order of integration,

$$
\left|\left\langle E_{k}^{n}(D) v, \psi\right\rangle\right|=\left|\int d \mu_{v}\left\langle\chi_{x}, E_{k}^{n}(-D) \psi\right\rangle\right| \leq C\|v\|_{\mathrm{TV}},
$$

which proves the result by the converse to Hölder's inequality.

\section{EXPERIMENTS, EXAMPLES AND DISCUSSION}

In this section, we present the results of some numerical experiments and examples which illustrate some of our results. 
A. A computation suggested by Theorem 1.1. The following experiment shows initial data bounded in $\mathscr{B}$ for which the Crank-Nicolson scheme for $u_{t}+u_{x}=0$ yields unbounded approximants as $h \rightarrow 0$ while $t$ and $\lambda$ remain fixed. For $k$ and $n$ fixed, $E_{k}^{n}(D)$, being smooth and having a periodic symbol, is a bounded operator from $L^{\infty}(\mathbf{R})$ into itself. In particular, Theorem 5.3.1 of Brenner et al. [6] shows that

$$
\left\|E_{k}^{n}(D)\right\|_{L^{\infty}(\mathbf{R}) \rightarrow L^{\infty}(\mathbf{R})}=\mathrm{O}\left(n^{1 / 2}\right) .
$$

On the other hand, the dispersive character of the Crank-Nicolson scheme yields the inequality

$$
\left\|E_{k}^{n}(D)\right\|_{L^{\infty}(\mathbf{R}) \rightarrow L^{\infty}(\mathbf{R})} \geq c n^{1 / 2} .
$$

Trefethen presents a nice explanation of this in $[23,24]$. He argues that a carefully constructed spike function $v_{h}(x)$ with height 1 and width $\mathrm{O}(1 / h)$ is sent by the backward Crank-Nicolson scheme with symbol

$$
E_{k}^{n}(-D)=E_{k}^{n}(D)^{*}=E_{k}^{n}(D)^{-1}
$$

(note that $E_{k}^{n}(D)$ is $L^{2}(\mathbf{R})$ unitary) to a wave packet that is uniformly distributed over an interval of width $\mathrm{O}(t)$. Thus, there is an increase in width of $\mathrm{O}(n)$, and since the $L^{2}(\mathbf{R})$ norm is preserved, the height suffers a decrease of $\mathrm{O}\left(n^{-1 / 2}\right)$. Taking the resulting functions as initial data for the forward scheme yields (5.1).

We have to be a little more careful in order to show that

$$
\left\|E_{k}^{n}(D)\right\|_{\mathscr{B} \rightarrow L^{\infty}(\mathbf{R})} \rightarrow \infty \text {. }
$$

Start with the simple spike function

$$
v_{h}(x):= \begin{cases}x / h+1, & -h \leq x \leq 0, \\ -x / h+1, & 0<x \leq h, \\ 0, & |x|>h,\end{cases}
$$

with (ignoring multiplicative constants)

$$
\hat{v}_{h}(\xi)=\frac{1-\cos (h \xi)}{h \xi^{2}} .
$$

It is easy to verify that $v_{h}$ and $E_{k}^{n}(-D) v_{h}$ are bounded below in the $\mathrm{B}_{2}^{1 / 2, \infty}$ norm, and so in the $\mathscr{B}$ norm, independently of $h$. Thus, taking the functions $E_{k}^{n}(-D) v_{h}$ as initial data does not suffice to show that

$$
\left\|E_{k}^{n}(D)\right\|_{\mathscr{B} \rightarrow L^{\infty}(\mathbf{R})}
$$

is unbounded as $k \rightarrow 0$.

To get around this, truncate the Fourier transform of $v_{h}$ by setting $w_{h}(x):=$ $\mathscr{F}^{-1}\left(\hat{v}_{h}(\xi) \chi(\xi)\right), \chi(\xi)=\chi(\xi, h), \chi \in C^{\infty}(\mathbf{R}) \times C^{\infty}(\mathbf{R}), \chi(\xi) \equiv 1$ for $|\xi| \leq$ $1 / h^{.5}$ and $\chi(\xi) \equiv 0$ for $|\xi|>1 / h^{.5}+\varepsilon$, for some $\varepsilon>0$. It is easy to see that

$$
\left\|E_{k}^{n}(-D) w_{h}\right\|_{\mathrm{B}_{2}^{1 / 2, \infty}}=\left\|w_{h}\right\|_{\mathrm{B}_{2}^{1 / 2, \infty}}=\mathrm{O}\left(h^{1 / 2}\right)
$$




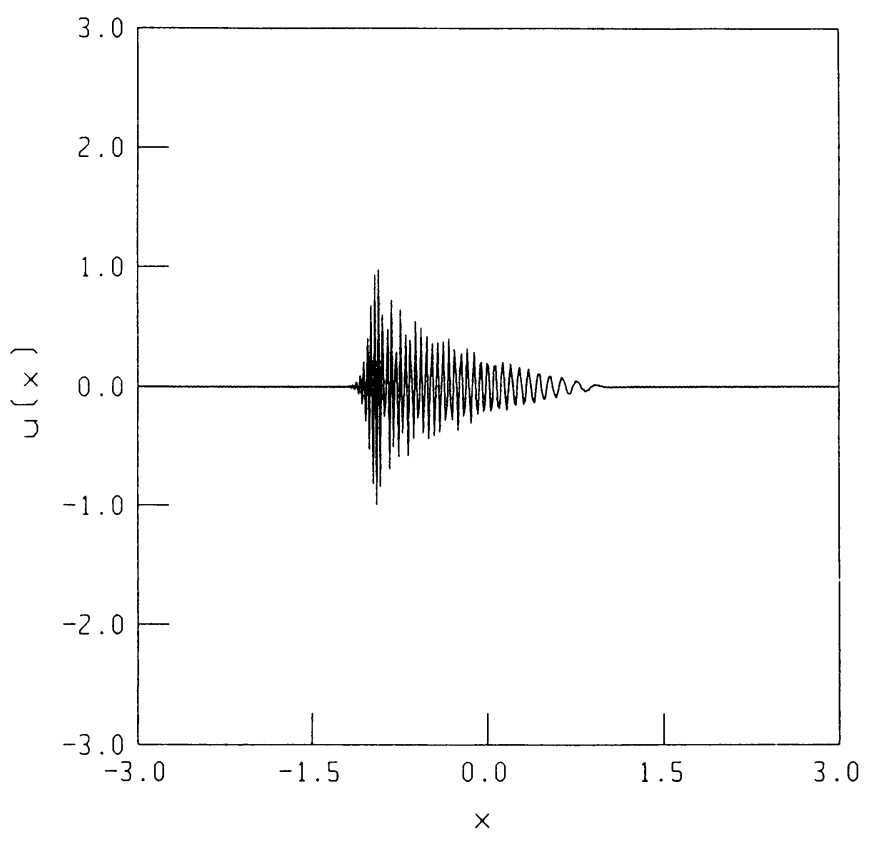

FIGURE 5.1

Plot of the initial data $u_{h_{3}}$.

$$
h=2^{-7}, \quad \lambda=.5, \quad t=0 \text {. }
$$

as $h \rightarrow 0$. Next, set

$$
u_{h}(x):=\frac{E_{k}^{n}(-D) w_{h}(x)}{\left\|E_{k}^{n}(-D) w_{h}(x)\right\|_{L^{\infty}(\mathbf{R})}} .
$$

$u_{h}$ is bounded in $\mathscr{B}$ independently of $h$, and since $\left\|E_{k}^{n}(-D) w_{h}\right\|_{L^{\infty}(\mathbf{R})}=$ $\mathrm{O}\left(h^{1 / 2}\right)$, it follows that

$$
\left\|u_{h}(x)\right\|_{\mathscr{B}}=1+\mathrm{O}\left(h^{1 / 2}\right) .
$$

Therefore, $u_{h}$ will produce the desired behavior in the approximants.

Unfortunately, $u_{h}(x)$ is not the regularly shaped wave packet (see Figure 5.1) that Trefethen's argument requires. Thus, we might expect that the forward Crank-Nicolson scheme used on the data $u_{h}$ produces a height increase of only $\mathrm{O}\left(n^{\alpha}\right)$ for some $\alpha<1 / 2$. This appears to be the case. For the numerical experiments, we approximate the integrals defining $w_{h}(x)$ for $h=2^{-4-i}, i=$ $1,2, \ldots, 6$, by the fast Fourier transform, and use the result to compute $u_{h_{i}}$ by the formula above. We use the Crank-Nicolson scheme with periodic boundary conditions at $x= \pm 2$.

Figures 5.1 and 5.2 contain plots of the functions $u_{h_{3}}$ and $E_{k_{3}}^{n}(D) u_{h_{3}}$ versus $x$ computed at time $t=n k$. The focussing of the wave train into a spike is clear. 


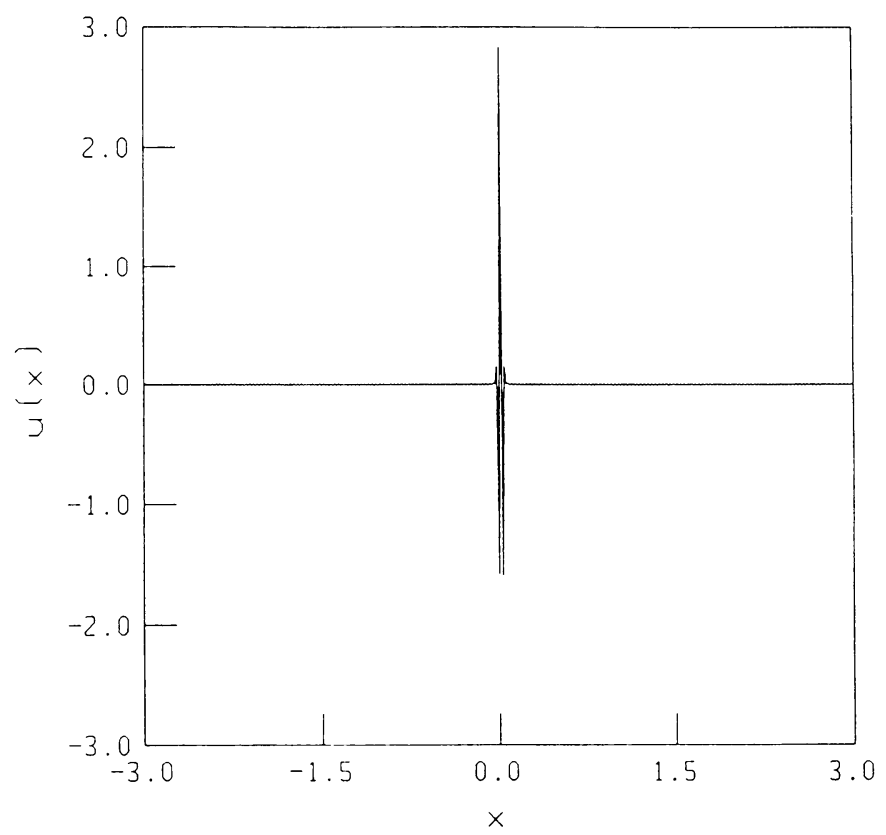

FIGURE 5.2

Plot of the Crank-Nicolson approximant computed on the initial data $u_{h_{3}}$.

$$
h=2^{-7}, \quad \lambda=.5, \quad t=1 .
$$

Since we expect that

$$
\left\|E_{k_{i}}^{n}(D) u_{h_{i}}\right\|_{l^{\infty}}=\mathrm{O}\left(h_{i}^{-\alpha}\right),
$$

we have computed a least squares line fit using as data the log of the ratio of the maximum heights of the functions to the height of the function with the largest step size $2^{-5}$ versus the log of the ratio of the corresponding steps. The result suggests that $\alpha \sim .331$ with a correlation factor of .994 . See Figure 5.3.

It is clear how to construct such a set of functions for some other purely dispersive scheme. Partially dissipative schemes like the Lax-Wendroff schemes are another matter, however. The higher-order dissipative effect limits the range of $x$ over which dispersion is dominant. However, it can be shown that this range increases in size as $h$ tends to zero, and this fact can be used to construct examples which show unbounded behavior as for the Crank-Nicolson scheme.

B. Experiments suggested by Theorem 1.2 and the material in $\$ 2$. Next, we present three experiments which suggest the usefulness of the normal form for predicting the behavior of the difference scheme. In particular, for the CrankNicolson scheme discussed above, we use the normal form to give estimates of: (1) the speed at which the largest peak moves toward the position of the discontinuity as $h \rightarrow 0$; (2) the limiting height of the approximants; and (3) the height of the approximate solution at the point of discontinuity. 


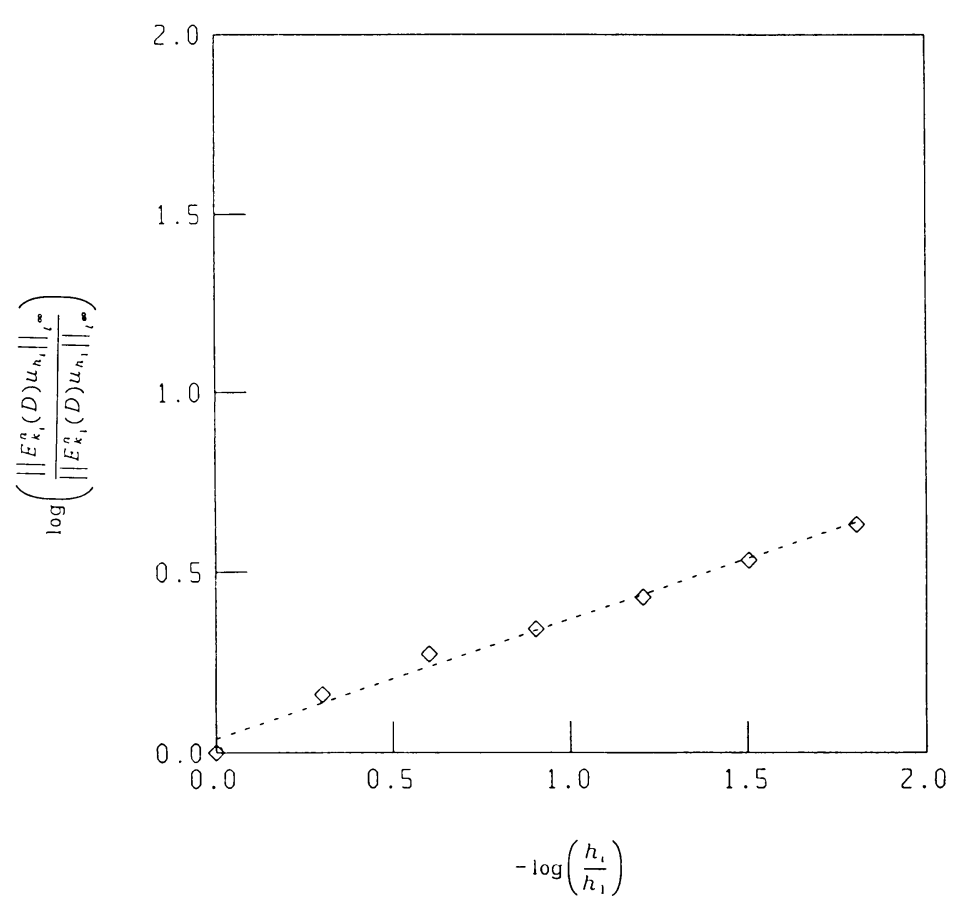

FIGURE 5.3

Plot relating the logarithm of the increase in height of the approximant from $t=0$ to $t=1$ to the logarithm of the step size. The diamonds represent the measured points. The dashes represent the least squares fitted line.

We use Theorem 2.5 as the basis for our predictions. By that theorem, if $x-1=\mathrm{O}\left(h^{2 / 3}\right)$, then in the limit as $h \rightarrow 0$ the difference scheme approximant will differ significantly from the true solution. The first result we present in Figure 5.4 compares the distance of the largest peak to the position of the discontinuity of the true solution to the step size. Since we expect that the distance goes like $a h^{\beta}$, we plot the log of the distance versus the log of the step size for $h=.2, .1, .08, \ldots, .02, .01, .008, \ldots, .002$. We have computed a least squares line fit for this data and found that $|a| \approx 1.95$ and $\beta \approx .67$ with a correlation of .998 . Note that the true peak can be located only to an accuracy of $\pm h$ for a given step size.

From Theorem 2.5, the limiting height of points which satisfy $(x-1)=a h^{2 / 3}$ will be given by the integral

$$
\mathscr{A}(c, a):=\frac{2}{\pi} \int_{0}^{\infty} \frac{\sin \left(c a \xi+\xi^{3}\right)}{\xi} d \xi,
$$

where $c$ is a constant generated in the construction of the normal form. If we consider $\mathscr{A}$ as a function of $a c$, then it is equal to a primitive of the Airy function $\operatorname{Ai}(x):=\mathscr{F}^{-1}\left(e^{i \xi^{3} / 3}\right)$ evaluated at $a c$, and therefore the maxima and minima of $\mathscr{A}$ occur at the values of $a c$ which are zeros of the Airy function. 


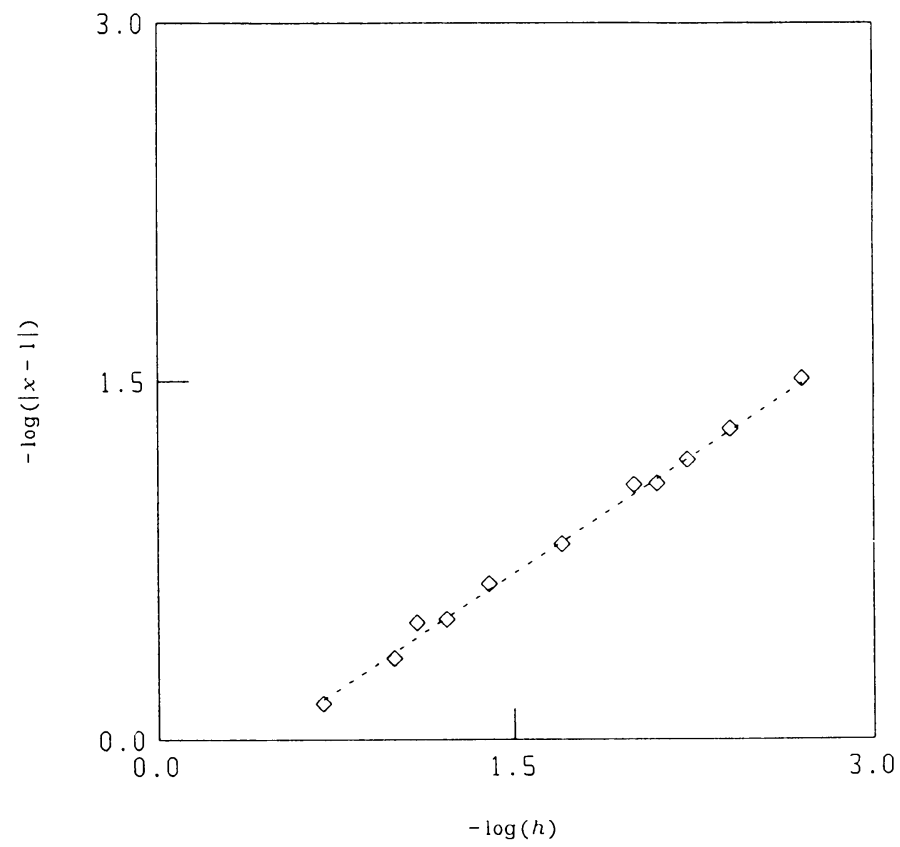

FIGURE 5.4

Plot relating the logarithm of the distance of the largest peak of the approximant to the position $x=1$ to the logarithm of the step size. The diamonds represent the measured values. The dashes represent the least squares fitted line.

These zeros are located along the negative part of the axis at well-spaced intervals (see [15]), and therefore the oscillations in the approximant located on a scale of $h^{2 / 3}$ will be more or less regularly spaced and their limiting height can be obtained from $\mathscr{A}$.

When the symbol is analytic (which is true of the difference schemes considered), one can construct a normal form which is obtained by an analytic map and which is unique. In the $C^{\infty}$ category, though the normal form is not unique, the coefficients $B_{j}(y)$ do have unique Taylor series and thus the constant $c$ above is uniquely determined. In the analytic case, the normal form can be constructed by different means (see Arnol'd [3]); the alternative method yields precise information about the final normal form immediately. For example, the symbol of the Crank-Nicolson operator has the Taylor expansion

$$
E_{k}(\xi)=e^{i\left(-\frac{1}{2} h \xi+\frac{7}{32} h^{3} \xi^{3}+\cdots\right)} \text { for } \lambda=\frac{1}{2},
$$

and the relevant part of the analytic normal form on the scale of $h^{2 / 3}$ is given by $e^{-i a \xi+i \frac{7}{32} \xi^{3}}$. This gives $c=-\left(\frac{32}{7}\right)^{1 / 3}$.

We substituted the values $a=-1.95$ measured directly from the approximants computed above and this $c$ into (5.2) and then approximated the integral using Romberg integration over a range of $[0,10]$ and obtained the number -1.54 . (We checked that the truncated range was sufficiently large by comput- 
ing over intervals with right-hand points ranging from 5 to 10 and obtained the same result to three places each time.) Below, we list the heights at the peaks for the values of $h$ used to determine the speed of the peaks:

\begin{tabular}{|c|c|}
\hline step size $h$ & $\begin{array}{c}\text { height of the largest } \\
\text { peak }\end{array}$ \\
\hline .2 & -1.64 \\
.1 & -1.60 \\
.08 & -1.56 \\
.06 & -1.58 \\
.04 & -1.58 \\
.02 & -1.55 \\
.01 & -1.55 \\
.008 & -1.55 \\
.006 & -1.55 \\
.004 & -1.55 \\
.002 & -1.55 \\
\hline
\end{tabular}

The data support the conjecture that if $x_{n}$ is the measured value of the location of the largest peak of the approximant and $a$ comes from the analytic normal form then

$$
\frac{x_{n}-1}{a h^{2 / 3}} \rightarrow 1, \quad n \rightarrow \infty .
$$

Another prediction that is easy to make is the height at which the approximant crosses the point of discontinuity. We take the limit as $a \rightarrow 0$ from above in Theorem 2.5 and are left with computing the oscillatory integral

$$
\frac{1}{\pi} \text { P.V. } \int e^{i \xi^{3}} \frac{d \xi}{\xi}=\frac{2}{\pi} \int_{0}^{\infty} \frac{\sin \left(\xi^{3}\right)}{\xi} d \xi=\frac{1}{3} \frac{2}{\pi} \int_{0}^{\infty} \frac{\sin (\eta)}{\eta} d \eta=\frac{1}{3} .
$$

The last equality is true because the integral on the right is just the value of the limit of the translated step function as $x \downarrow 1$. For comparison, we present a chart of the crossing points for the approximants computed above.

\begin{tabular}{|c|c|}
\hline step size $h$ & height at $x=1$ \\
\hline .2 & .36 \\
.1 & .35 \\
.08 & .35 \\
.06 & .35 \\
.04 & .18 \\
.02 & .22 \\
.01 & .24 \\
.008 & .34 \\
.006 & .34 \\
.004 & .34 \\
.002 & .33 \\
\hline
\end{tabular}




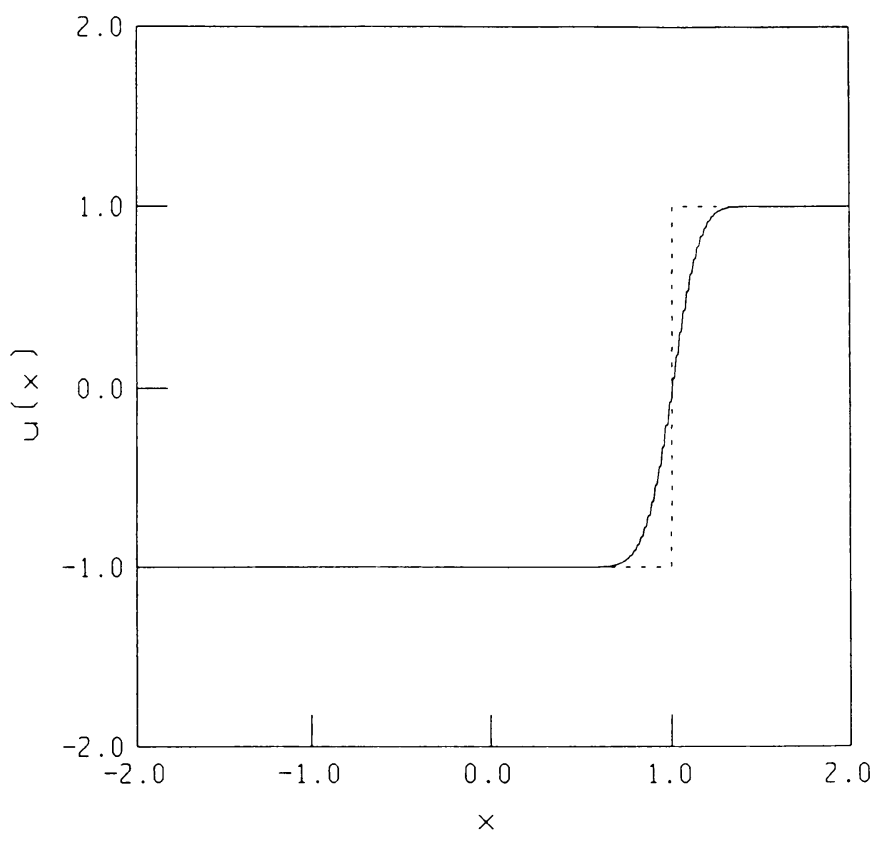

FIGURE 5.5

Plot of the Lax-Friedrichs approximant computed on step data at $t=1 . k=.01, \lambda=.5$. The dashes show the position of the true solution. The solid curve connects the points of the approximant.

Again, there seems to be good agreement between these data and the predicted height. (As for all of the results, the actual location of the crossing point is determined only to within $\pm h$, and all of the values above fall well into the range of heights associated with this error.)

C. Some examples of difference schemes. Finally, we present some examples of difference schemes.

Example 5.1. The Lax-Friedrichs scheme for $u_{t}+u_{x}=0$ is given by

$$
\begin{gathered}
\frac{U((n+1) k, j h)-(U(n k,(j+1) h)+U(n k,(j-1) h)) / 2}{k} \\
+\frac{U(n k,(j+1) h)-U(n k,(j-1) h)}{2 h}=0 .
\end{gathered}
$$

The corresponding time stepping operator is

$$
E_{k}=\frac{(1-\lambda)}{2} \tau_{-h}+\frac{(1+\lambda)}{2} \tau_{h}, \quad \text { with } \lambda:=\frac{k}{h} .
$$

The operator $E_{k}$ of the Lax-Friedrichs scheme is a contraction in $L^{p}(\mathbf{R})$, for $1 \leq p \leq \infty$, provided the Courant-Friedrichs-Lewy (CFL) condition $k / h \leq 1$ is satisfied. The associated maximum principle is visible in the results of a computation presented in Figure 5.5 with $k=.01$ and $\lambda=.5$. 


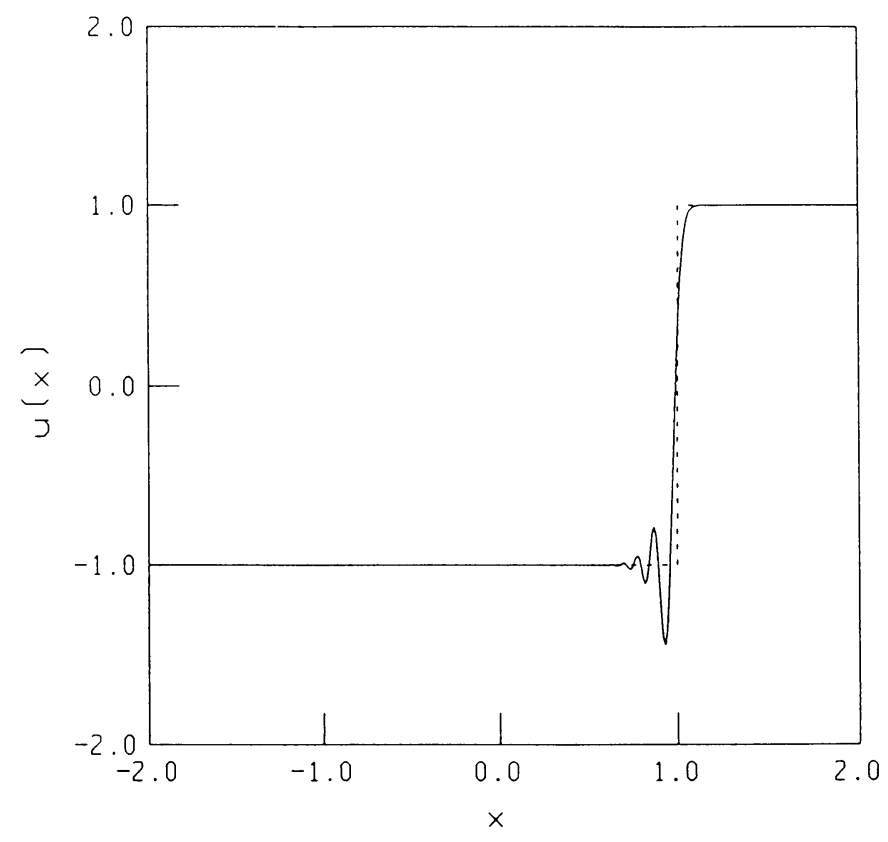

FIGURE 5.6

Plot of the Lax-Wendroff approximant computed on step data at $t=1 . k=.01, \lambda=.5$. The dashes show the position of the true solution. The solid curve connects the points of the approximant.

Example 5.2. The Lax-Wendroff scheme for $u_{t}+u_{x}=0$ is given by

$$
\begin{gathered}
\frac{U((n+1) k, j h)-U(n k, j h)}{k}+\frac{U(n k,(j+1) h)-U(n k,(j-1) h)}{2 h} \\
=\frac{k}{2} \frac{U(n k,(j+1) h)-2 U(n k, j h)+U(n k,(j-1) h)}{h^{2}}
\end{gathered}
$$

and its operator is

$$
E_{k}=\left(1-\lambda^{2}\right) I+\frac{\lambda(\lambda-1)}{2} \tau_{-h}+\frac{\lambda(\lambda+1)}{2} \tau_{h} .
$$

The symbol of the Lax-Wendroff scheme is

$$
E_{k}(\xi)=1-\lambda^{2}(1-\cos (\xi h))-i \lambda \sin (\xi h) .
$$

The Lax-Wendroff scheme is stable in $L^{2}(\mathbf{R})$ provided $\lambda \leq 1$. If $\lambda$ is assumed constant, then it can be shown that $E_{k}(D)$ is stable in $L^{\bar{p}}(\mathbf{R})$ only for $p=2$. In Figure 5.6, we use the Lax-Wendroff scheme on a single step with $k=.01$ and $\lambda=.5$.

Example 5.3. The following schemes are examples of methods which have a nonlinear relationship between $h$ and $k$. First, if we take the equation $u_{t}+$ $u_{x}=0$, and apply the difference scheme which uses a forward time difference 
and a centered $x$ difference:

$$
\frac{U((n+1) k, j h)-U(n k, j h)}{k}+\frac{U(n k,(j+1) h)-U(n k,(j-1) h)}{2 h}=0,
$$

then its symbol is $E_{k}(\xi)=1-i \frac{k}{h} \sin (\xi h)$. A simple computation shows that we need to assume that $k \leq c h^{2}$ to have $L^{2}(\mathbf{R})$ stability. For this scheme, with $k=h^{2}$, the expansion at $\xi_{0}=0$ is

$$
E_{k}(\xi)=\exp \left\{-i \xi k+\frac{i}{6} \xi^{3} k^{2}+k \rho_{0}(k, \xi)\right\},
$$

where $\rho_{0}(k, x)$ satisfies the hypotheses of Theorem 3.1. Another example: in the Crank-Nicolson scheme, we can take $k \gg h$, say $k=h^{1 / 2}$, and still have $L^{2}(\mathbf{R})$ stability. The expansion at $\xi_{0}=0$ for this method is

$$
E_{k}(\xi)=\exp \left\{-i \xi k+\frac{i}{12} \xi^{3} k^{3}+k \rho_{0}(k, \xi)\right\},
$$

where $\rho_{0}(k, \xi)$ satisfies the hypotheses of Theorem 3.1.

All computations were performed on an IBM PS/2 50 with a math coprocessor using Microsoft FORTRAN 4.0, HGRAPH graphics library and MATHCAD 2.0 .

\section{APPENDIX A. REDUCTION TO NORMAL FORM}

In this section, we prove the main technical result which is used to bring the phase function into a simple form called the normal form. We follow the proof given in Hörmander [15].

Theorem A1. Let $F(\xi, y)=\xi f\left(\xi^{2}, y\right)$, where $f(\eta, y)$ is $C^{\infty}$ in a neighborhood of the origin in $\mathbf{R} \times \mathbf{R}$. Assume that

$$
f(\eta, 0)=\eta^{\nu} f(\eta),
$$

where $f(0)>0$. Then there exist functions $\Xi(\eta, y)$ and $B_{j}(y), j=0, \ldots$, $\nu-1, C^{\infty}$ in a neighborhood of the origin, such that

$$
F(\xi, y)=\Xi^{2 \nu+1}+\sum_{j=0}^{\nu-1} B_{j}(y) \Xi^{2 j+1},
$$

with the properties that $B_{j}(y)=\mathrm{O}(y)$ as $y \rightarrow 0, \Xi(\xi, y)$ is odd in $\xi$, i.e., $\Xi(-\xi, y)=-\Xi(\xi, y)$, and furthermore

$$
\Xi(0,0)=0 \text { and } \frac{\partial \Xi}{\partial \xi}(0,0)>0 .
$$

Remark. This theorem was proved for the analytic case by Levinson [17], Chester et al. [7] and Arnol'd [2,3]. The above result is a consequence of the Malgrange Division Theorem and is due essentially to Mather (see [15, 28]). We state the Malgrange Division Theorem without proof. 
Theorem A2 (Malgrange Division Theorem). Let $h(\eta, y)$ be a real-valued $C^{\infty}$ function in a neighborhood of zero in $\mathbf{R} \times \mathbf{R}^{n}$. Assume that

$$
h(0,0)=\frac{\partial h}{\partial \eta}(0,0)=\cdots=\frac{\partial^{\nu-1} h}{\partial \eta^{\nu-1}}(0,0)=0 \text { and } \frac{\partial^{\nu} h}{\partial \eta^{\nu}} \neq 0 .
$$

Let $g(\eta, y)$ be any real $C^{\infty}$ function. Then there exist functions $q(\eta, y)$, $p_{j}(y), j=1, \ldots, \nu-1$, all $C^{\infty}$ in a neighborhood of zero, such that

$$
g(\eta, y)=q(\eta, y) h(\eta, y)+\sum_{j=0}^{\nu-1} p_{j}(y) \eta^{j}
$$

Corollary A3. Let $H(\xi, y)=h\left(\xi^{2}, y\right)$, where $h$ satisfies the assumptions of the theorem above. Let $G(\xi, y)=\xi g\left(\xi^{2}, y\right)$, where $g$ can be any $C^{\infty}$ function. Then there are functions $Q(\xi, y)=\xi q\left(\xi^{2}, y\right)$ and $p_{j}(y), j=0, \ldots, \nu-1$, all $C^{\infty}$, such that

$$
G(\xi, y)=Q(\xi, y) H(\xi, y)+\sum_{j=0}^{\nu-1} p_{j}(y) \xi^{2 j+1}
$$

Remark. The main interest of this corollary is that $Q(\xi, y)$ is odd in $\xi$.

Proof of Corollary A3. An application of Theorem A2 to the pair of functions $g(\eta, y)$ and $h(\eta, y)$ yields

$$
g(\eta, y)=q(\eta, y) h(\eta, y)+\sum_{j=0}^{\nu-1} p_{j}(y) \eta^{j}
$$

for $C^{\infty}$ functions $q$ and $p_{j}, j=0, \ldots, \nu-1$. Replacing $\eta$ by $\xi^{2}$ and multiplying by $\xi$ yields the result.

Proof of Theorem A1. Assume that $f(\eta, 0)=\eta^{\nu}$, i.e., $F(\xi, 0)=\xi^{2 \nu+1}$; this can be arranged by a smooth change of variables. The aim is to find functions $\xi(y)$ and $\mathbf{b}(y) \quad\left(\mathbf{b}:=\left(b_{0}, \ldots, b_{\nu-1}\right)\right)$ of $y$ in a neighborhood of zero, such that

$$
F(\xi, y)+\sum_{j=0}^{\nu-1} b_{j} \xi^{2 j+1}
$$

is constant. To construct them, differentiate $(\mathrm{A}-1)$ with respect to $y$ to get

$$
\left(F_{\xi}+\sum_{j=0}^{\nu-1}(2 j+1) b_{j} \xi^{2 j}\right) \xi^{\prime}+F_{y}+\sum_{j=0}^{\nu-1} b_{j}^{\prime} \xi^{2 j+1}
$$

(the prime denotes differentiation with respect to $y$ ). By assumption, the first bracket is an even function of $\xi$, i.e., of the form $h\left(\xi^{2}, y, \mathbf{b}\right)$, for some smooth $h$. Likewise, $F_{y}=\xi g\left(\xi^{2}, y, \mathbf{b}\right)$ for some smooth $g$. Applying Corollary 
A3 to this pair of functions, we find $Q(\xi, y, \mathbf{b})$ smooth and $p_{j}(y, \mathbf{b}), j=$ $0, \ldots, \nu-1$, smooth such that

$$
F_{y}=Q \cdot\left(F_{\xi}+\sum_{j=0}^{\nu-1}(2 j+1) b_{j} \xi^{2 j}\right)+\sum_{j=0}^{\nu-1} p_{j}(y, \mathbf{b}) \xi^{2 j+1},
$$

where $Q$ is odd in $\xi: Q(-\xi, y, \mathbf{b})=-Q(\xi, y, \mathbf{b})$. Inserting this formula into (A-2), the desideratum is achieved, provided that $\xi$ and $\mathbf{b}$ are chosen to be solutions of the differential equation

$$
\xi^{\prime}=-Q(\xi, y, \mathbf{b}), \quad \mathbf{b}^{\prime}=-\mathbf{p}(y, \mathbf{b})
$$

Since $Q$ and $\mathbf{p}$ are smooth in a neighborhood of the origin, there exist smooth solutions $\xi(\Xi, y, \mathbf{B})$ and $\mathbf{b}(\mathbf{B}, y)$ having $\Xi$ resp. $\mathbf{B}$ as initial data, i.e.,

$$
\xi(\Xi, 0, \mathbf{B})=\Xi \text { and } \mathbf{b}(\mathbf{B}, 0)=\mathbf{B} \text {. }
$$

By $(A-3), \frac{\partial \mathbf{b}}{\partial \mathbf{B}}(\mathbf{B}, 0)=1$. Therefore, by the implicit function theorem, there exists a smooth function $\mathbf{B}(y)$ such that

$$
\mathbf{b}(\mathbf{B}(y), y)=0
$$

for $y$ in a neighborhood of zero. Obviously, $\mathbf{B}(0)=0$. This, together with (A-1), yields

$$
F(\xi(\Xi, y, \mathbf{B}(y)), y)=\Xi^{2 \nu+1}+\sum_{j=0}^{\nu+1} \mathbf{B}_{j}(y) \Xi^{2 j+1} .
$$

We also used that $F(\Xi, 0)=\Xi^{2 \nu+1}$.

Finally observe that by (A-3), again using the implicit function theorem, $\xi(\Xi, y, \mathbf{B}(y))$ can be inverted to yield $\Xi(\xi, y, \mathbf{B}(y))$, depending smoothly on $y$ in a neighborhood of zero. Since $Q$ is odd in $\xi,-\xi(-\Xi, y, \mathbf{B})$ is also a solution with the same initial data and hence $\xi(-\Xi, y, \mathbf{B})=-\xi(\Xi, y, \mathbf{B})$ in the whole neighborhood of existence. Therefore,

$$
\Xi(-\xi, y, \mathbf{B}(y))=-\Xi(\xi, y, \mathbf{B}(y)) \text {. }
$$

Since $\frac{\partial \xi}{\partial \Xi}(\Xi, 0,0)=1, \frac{\partial \Xi}{\partial \xi}(0,0,0)=1$ as well; this, together with $(\mathrm{A}-5)$, yields the desired result.

\section{ACKNOWLEDGMENT}

We thank Stig Larsson, John Mather and David Stewart for helpful discussions.

\section{BIBLIOGRAPHY}

1 M. Y. T. Apelkrans, On difference schemes for hyperbolic equations with discontinuous initial values, Math. Comp. 22 (1968), 525-539.

2. V. I. Arnol'd, Singularities of smooth mappings, Russian Math. Surveys 23 (1968), 1-43.

3. __ Singularity theory. Cambridge Univ. Press, New York. 1981. 
4. P. Brenner and V. Thomée, Stability and convergence rates in $L^{p}$ for certain difference schemes, Math. Scand. 27 (1970), 5-23.

5. __ Estimates near discontinuities for some difference schemes, Math. Scand. 28 (1971), 329-340.

6. P. Brenner, V. Thomée, and L. Wahlbin, Besov spaces and applications to difference methods for initial value problems, Springer-Verlag, New York, 1975.

7. C. Chester, B. Friedman, and F. Ursell, An extension of the method of steepest descents, Proc. Cambridge Philos. Soc. 53 (1957), 599-611.

8. R. C. Y. Chin, Dispersion and Gibbs phenomenon associated with difference approximations to initial boundary-value problems for hyperbolic equations, J. Comput. Phys. 18 (1975), 233-247.

9. R. C. Y. Chin and G. W. Hedstrom, A dispersion analysis for difference schemes: Tables of generalized Airy functions, Math. Comp. 32 (1978), 1163-1170.

10. D. J. Estep, $L^{\infty}$ bounds for families of translation invariant, $L^{2}$ stable operators in one dimension, Ph.D. thesis, The University of Michigan, 1987.

11. M. Gollubitsky and V. Guillemin, Stable mappings and their singularities, Springer, New York, 1973.

12. G. W. Hedstrom, The near-stability of the Lax-Wendroff method, Numer. Math. 7 (1965), 73-77.

13. 363-406.

14. L. Hörmander, Estimates for translation invariant operators in $L^{p}$ spaces, Acta. Math. 104 (1960), 93-140.

15. __ Linear partial differential equations, vol. 1, Springer-Verlag, New York, 1983.

16. F. John, Partial differential equations, 4th ed., Springer-Verlag, New York, 1982.

17. N. Levinson, Transformation of an analytic function of several variables to a canonical form, Duke Math. J. 28 (1961), 345-353.

18. J. Peetre and V. Thomée, On the rate of convergence for discrete initial value problems, Math. Scand. 21 (1967), 158-176.

19. R. D. Richtmyer and K. W. Morton, Difference methods for initial-value problems, 2nd ed., Interscience, New York, 1967.

20. W. Rudin, Real and complex analysis, McGraw-Hill, 1974.

21. E. M. Stein, Oscillatory integrals in Fourier analysis, in Beijing Lectures in Harmonic Analysis (E. M. Stein, ed.), Princeton Univ. Press, Princeton, N.J., 1986, pp. 307-355.

22. V. Thomée, On the rate of convergence of difference schemes for hyperbolic equations, Numerical Solution of Partial Differential Equations. II (B. Hubbard, ed.), Academic Press, New York, 1971, pp. 585-622.

23. L. N. Trefethen, Group velocity in finite difference schemes, SIAM Rev. 24 (1982), pp. 113-136.

24. __ Dispersion, dissipation and stability, Numerical Analysis (D. F. Griffiths and G. A. Watson, eds.), Longman, 1986.

25. S. Wainger, Averages and singular integrals over lower dimensional sets, Beijing Lectures in Fourier Analysis (E. M. Stein, ed.), Princeton Univ. Press, Princeton, N.J., 1986, pp. 357-421.

26. G. B. Whitham, Linear and nonlinear waves, Wiley, New York, 1974.

27. K. Yosida, Functional analysis, 6th ed., Springer-Verlag, New York, 1982.

28. E. C. Zeeman, Catastrophe theory: Selected papers, Addison-Wesley, Reading, Mass., 1977.

School of Mathematics, Georgia Institute of Technology, Atlanta, Georgia 30332 (Estep and Loss).E-mail: estep@math.gatech.edu; loss@math.gatech.edu

Department of Mathematics, University of Michigan, Ann Arbor, Michigan 48109 (Rauch).E-mail: jeffrey_rauch@ub.cc.umich.edu 